UCLA $/ 07 /$ TEP $/ 16$

SLAC-PUB-12609

\title{
Unexpected Cancellations in Gravity Theories
}

\author{
Z. Bern ${ }^{a}$, J. J. Carrasco ${ }^{a}$, D. Forde ${ }^{a, b}$, H. Ita ${ }^{c}$ and H. Johansson ${ }^{a}$ \\ ${ }^{a}$ Department of Physics and Astronomy, \\ UCLA, Los Angeles, CA 90095-1547, USA \\ ${ }^{b}$ Stanford Linear Accelerator Center, \\ Stanford University, Stanford, CA 94309, USA \\ ${ }^{c}$ Department of Physics, Swansea University, Swansea, SA2 8PP, UK
}

(Dated: July, 2007)

\begin{abstract}
Recent computations of scattering amplitudes show that $\mathcal{N}=8$ supergravity is surprisingly well behaved in the ultraviolet and may even be ultraviolet finite in perturbation theory. The novel cancellations necessary for ultraviolet finiteness first appear at one loop in the guise of the "notriangle hypothesis". We study one-loop amplitudes in pure Einstein gravity and point out the existence of cancellations similar to those found previously in $\mathcal{N}=8$ supergravity. These cancellations go beyond those found in the one-loop effective action. Using unitarity, this suggests that generic theories of quantum gravity based on the Einstein-Hilbert action may be better behaved in the ultraviolet at higher loops than suggested by naive power counting, though without additional (supersymmetric) cancellations they diverge. We comment on future studies that should be performed to support this proposal.
\end{abstract}

PACS numbers: 11.15.Bt, 11.25.Db, 11.25.Tq, 11.55.Bq, 12.38.Bx 


\section{INTRODUCTION}

Recent calculations of four-point scattering amplitudes in the maximally supersymmetric gravity theory of Cremmer and Julia [1] indicate the existence of novel ultraviolet cancellations at three and higher loops, which may even lead to the perturbative finiteness of the theory [2, 3]. String dualities have also been used to argue for ultraviolet finiteness of $\mathcal{N}=8$ supergravity [4], though difficulties with decoupling towers of massive states may alter this conclusion [5].

At one loop in the $\mathcal{N}=8$ theory, the corresponding novel cancellations are encapsulated in the "no-triangle hypothesis" [6 9]. In general, dimensionally regularized amplitudes in four dimensions can be expressed as a linear combination of scalar box, triangle and bubble integrals together with rational terms [10, 11]. The no-triangle hypothesis states that all one-loop amplitudes in $\mathcal{N}=8$ supergravity can be expressed solely in terms of scalar box integrals and that neither triangle integrals, bubble integrals nor additional rational terms appear. This hypothesis is surprising from the point of view of power counting, which together with standard integral reduction formulas - would imply that triangle integrals should appear beginning at five points and bubble integrals at six points. The cancellation of triangle and bubble integrals were first observed in maximally helicity violating (MHV) amplitudes of the $\mathcal{N}=8$ theory [6]. More recently, this observation was extended to the hypothesis that the same cancellations occur in all $\mathcal{N}=8$ one-loop amplitudes, so that they are expressed solely in terms of scalar box integrals [7]. It has been confirmed for six-graviton amplitudes, by explicit computation [9]. Furthermore, at seven points the infrared singularities have been shown to be consistent with the no-triangle hypothesis [8, 9]. Beyond six and seven points, scaling and factorization properties of the amplitudes provide strong evidence that the no-triangle hypothesis holds for the remaining amplitudes in $\mathcal{N}=8$ supergravity [7, 9]. In ref. [2], these one-loop cancellations were argued to lead to an improved ultraviolet behavior in classes of terms encountered in multi-loop calculations in the $\mathcal{N}=8$ theory. At three loops, the improvement in the ultraviolet behavior due to these and related cancellations has been confirmed by the explicit calculation of the complete four-point scattering amplitude in the $\mathcal{N}=8$ theory [3]. The consistency of the Regge limit with improved ultraviolet behavior in $\mathcal{N}=8$ supergravity has also been recently discussed in ref. [12]. 
Supersymmetry, in particular, has long been studied for its ability to reduce the degree of divergence of gravity theories [13]. However, all superspace power counting arguments ultimately delay the onset of divergences only by a finite number of loops, depending upon assumptions regarding the types of superspaces and invariants that can be constructed.

If the cancellations observed in the $\mathcal{N}=8$ theory are only partly due to supersymmetry, then what might account for the remainder of the observed cancellations? Here we propose that these extra cancellations are generic to any quantum gravity theory based on the Einstein-Hilbert action. These cancellations are not at all obvious in Feynman diagrams which individually obey a naive power counting. Rather, they will be manifest only in carefully chosen representations of the amplitudes. Our proposal is that in supersymmetric theories, the supersymmetric cancellations are on top of these cancellations. For the $\mathcal{N}=8$ theory at one loop it is the combination of cancellations that leads to the "no-triangle hypothesis". Following the same line of reasoning as used in the $\mathcal{N}=8$ theory [2], suggests that the observed one-loop cancellations in non-supersymmetric pure gravity will lead to a softening of the ultraviolet behavior at higher loops.

For non-supersymmetric theories these additional cancellations are in general insufficient to render the theory ultraviolet finite. Indeed, explicit calculations show that gravity coupled to matter generically diverges at one loop [15]. Pure Einstein gravity in four dimensions possesses a cancellation at one loop, distinct from the ones being discussed here, due to the absence of a viable counterterm, delaying the divergence to two loops. This two-loop divergence was established by Goroff and Sagnotti through direct computation of that divergence [16] and later confirmed by van de Ven [17].

Here, as a first step in checking the hypothesis that there are novel cancellations in nonsupersymmetric gravity theories, we will investigate pure gravity at one loop. To carry out our investigation of cancellations we make use of the unitarity method [14, 18, 19]. In order to observe the cancellations we reduce the amplitudes to combinations of box, bubble and triangle integrals. The cancellations then manifest themselves in unexpectedly low powers of loop momentum in these integrals. The reduction to the basic integrals allows us to combine the contributions coming from the higher-point integrals to make the cancellations explicit. We do so using powerful new loop integration methods [9, 20 24], based on generalized unitarity [25] and complex momenta [16, 26]. In particular, we make extensive use of the formalism introduced in ref. [24]. This will allow us to observe the hidden cancellations 
through simple scaling arguments.

The unitarity method requires as input tree amplitudes, which we construct via KawaiLewellen-Tye (KLT) relations [27, 28] and on-shell recursion relations [29-32]. For MHV tree amplitudes, we use the form obtained by Berends, Giele and Kuijf [33] via the KLT relations. This is a particularly compact form, making apparent various useful properties of the amplitudes.

The one-loop cancellations we observe rely on rather generic properties of gravity treelevel amplitudes. In particular, certain scaling properties of the tree amplitudes have to be independent of the number of scattering particles. These cancellations are quite reminiscent of ones that occur at tree level under the large complex deformations needed to prove the validity of on-shell recursion relations [31, 32]. Indeed, the connection of large complex deformations to the absence of scalar bubble integrals in $\mathcal{N}=8$ supergravity has been already

noted in ref. [9]. This connection suggests that the cancellations will be present in theories that are "fully constructible" from on-shell recursion relations, in the sense of ref. [34]. In such theories, the tree amplitudes can be obtained using on-shell recursion using only three vertices as the input. Remarkably, Einstein gravity is in this class.

This paper is organized as follows. In section II, we review properties of gravity tree amplitudes, including the cancellations that are present under large complex deformations. Then in section III we describe the methods we use to count the leading powers of loop momentum in the triangle and bubble integrals contributing in Einstein gravity. In this section we also contrast these cancellations against the well known one which renders pure Einstein gravity finite at one loop. We present the explicit power counting of the triangle and bubble integrals in section [V] In section $\mathrm{V}$ we explain the relationship between the cancellations we observe at one loop and ones that were observed previously at tree level. We also give a heuristic explanation of observed one-loop cancellations, based on universal factorization properties of one-loop amplitudes. We conclude and comment on the outlook in section $\mathrm{VI}$.

\section{PROPERTIES OF TREE AMPLITUDES}

In this section, we first review some well known representations of tree amplitudes, used later for explicit computations, and then review cancellations in tree amplitudes needed for 
on-shell recursion relations.

\section{A. Notation for gravity tree amplitudes}

To expose useful properties of scattering amplitudes in four dimensions we employ the spinor-helicity formalism [35, 36], with spinor products,

$$
\langle j l\rangle=\varepsilon^{\alpha \beta} \lambda_{j \alpha} \lambda_{l \beta}=\bar{u}_{-}\left(k_{j}\right) u_{+}\left(k_{l}\right), \quad[j l]=\varepsilon^{\dot{\alpha} \dot{\beta}} \tilde{\lambda}_{j \dot{\alpha}} \tilde{\lambda}_{l \dot{\beta}}=\bar{u}_{+}\left(k_{j}\right) u_{-}\left(k_{l}\right),
$$

where $u_{ \pm}(k)$ is a massless Weyl spinor with momentum $k$ and positive or negative chirality. It will also be convenient to use the bra-ket notation for the contractions of Weyl spinors; $\left\langle j^{\mp} \mid l^{ \pm}\right\rangle=\bar{u}_{\mp}\left(k_{j}\right) u_{ \pm}\left(k_{l}\right)$. With the normalizations used here, the spinor inner products are related to Lorentz inner products via, $\langle l j\rangle[j l]=\frac{1}{2} \operatorname{Tr}\left[k_{j} k_{l}\right]=2 k_{j} \cdot k_{l}=s_{j l}$. A useful identity is the Schouten identity,

$$
\langle 12\rangle\langle 34\rangle=\langle 23\rangle\langle 41\rangle+\langle 24\rangle\langle 13\rangle
$$

valid for four arbitrary spinors $\lambda_{1}, \lambda_{2}, \lambda_{3}$ and $\lambda_{4}$. In the spinor-helicity formalism gluon (spin 1) polarization vectors take the form,

$$
\varepsilon_{\mu}^{ \pm}\left(k_{i}, q_{i}\right)=\frac{1}{2} \frac{\left\langle q_{i}^{\mp}\left|\gamma_{\mu}\right| k_{i}^{\mp}\right\rangle}{\left\langle q_{i}^{\mp} \mid k_{i}^{\mp}\right\rangle},
$$

where $k_{i}$ is the momentum carried by the particle and $q_{i}$ is a null reference momentum. The graviton polarization is,

$$
\varepsilon_{\mu \nu}^{ \pm}\left(k_{i}, q_{i}\right)=\varepsilon_{\mu}^{ \pm}\left(k_{i}, q_{i}\right) \varepsilon_{\nu}^{ \pm}\left(k_{i}, q_{i}\right) .
$$

It is worth noting that the tracelessness condition $\varepsilon_{\mu}{ }^{\mu}=0$ is automatically enforced since $\left\langle q_{i}^{\mp}\left|\gamma_{\mu}\right| k_{i}^{\mp}\right\rangle$ is a complex null vector.

A particularly useful representation for gravity tree amplitudes is based on the Kawai, Lewellen and Tye relations [27] between open and closed string theory tree-level amplitudes, especially since this exposes the intimate connection between gauge and gravity amplitudes. In the low-energy limit these become relations between gravity and gauge theory amplitudes. Not only do they hold for any tree amplitudes obtained from the low-energy limit of a string theory, but they appear to hold more generally [28]. For three through six points the relations are,

$$
M_{3}^{\text {tree }}(1,2,3)=i A_{3}^{\text {tree }}(1,2,3) A_{4}^{\text {tree }}(1,3,2)
$$




$$
\begin{aligned}
M_{4}^{\text {tree }}(1,2,3,4)= & -i s_{12} A_{4}^{\text {tree }}(1,2,3,4) A_{4}^{\text {tree }}(1,2,4,3) \\
M_{5}^{\text {tree }}(1,2,3,4,5)= & i s_{12} s_{34} A_{5}^{\text {tree }}(1,2,3,4,5) A_{5}^{\text {tree }}(2,1,4,3,5) \\
& \quad+i s_{13} s_{24} A_{5}^{\text {tree }}(1,3,2,4,5) A_{5}^{\text {tree }}(3,1,4,2,5) \\
M_{6}^{\text {tree }}(1,2,3,4,5,6)= & -i s_{12} s_{45} A_{6}^{\text {tree }}(1,2,3,4,5,6)\left[s_{35} A_{6}^{\text {tree }}(2,1,5,3,4,6)\right. \\
& \left.\quad+\left(s_{34}+s_{35}\right) A_{6}^{\text {tree }}(2,1,5,4,3,6)\right]+\mathcal{P}(2,3,4) .
\end{aligned}
$$

Expressions for any numbers of legs may be found in appendix A of ref. [6]. Here the $M_{n}^{\text {tree' }}$ s are $n$-point tree-level amplitudes in a gravity theory. The $A_{n}^{\text {tree's }}$ are color-ordered gauge-theory partial amplitudes, with $A_{n}^{\text {tree }}(1,2, \ldots n)$ representing the kinematic coefficient of the color trace, $\operatorname{Tr}\left[T^{a_{1}} T^{a_{2}} \cdots T^{a_{n}}\right]$. In the six-point case, in eq. (2.8), $\mathcal{P}(2,3,4)$ signifies a sum over permutations over the legs 2,3 and 4 . We have suppressed factors of the coupling constants in the gauge theory and gravity amplitudes. The KLT relations are valid for any configuration of helicities and particles. As an abbreviation we use the labels " $1, \ldots, n$ " to denote the momenta $k_{1}, \ldots, k_{n}$ and polarizations or spinors of external legs.

For example, using known expressions for the gauge theory amplitudes [36, 37], for $n=$ 3, 4 the KLT relations give us,

$$
\begin{aligned}
M_{3}^{\text {tree }}\left(1^{-}, 2^{-}, 3^{+}\right) & =i \frac{\langle 12\rangle^{6}}{\langle 23\rangle^{2}\langle 31\rangle^{2}}, \\
M_{4}^{\text {tree }}\left(1^{-}, 2^{-}, 3^{+}, 4^{+}\right) & =i \frac{[34]\langle 12\rangle^{6}}{\langle 23\rangle\langle 34\rangle\langle 41\rangle\langle 24\rangle\langle 31\rangle} .
\end{aligned}
$$

Although very useful, the KLT form often makes all- $n$ analyses difficult due to large permutation sums and non-manifest factorization and scaling properties. For the specific case of MHV all- $n(n>4)$ graviton amplitudes, Berends, Giele and Kuijf (BGK) [33] presented the more manageable expression,

$$
\begin{aligned}
& M_{n}^{\text {tree }}\left(1^{+}, 2^{+}, \ldots, a^{-}, \ldots, b^{-}, \ldots, n^{+}\right)= \\
& \quad-i\langle a b\rangle^{8} \sum_{\mathcal{P}(3,4, \ldots, n-1)} \frac{\prod_{l=3}^{n-1}\left\langle n^{-}\left|K_{2 \ldots(l-1)}\right| l^{-}\right\rangle}{\prod_{i=1}^{n-2}\langle i(i+1)\rangle\langle 1(n-1)\rangle\langle 1 n\rangle^{2}\langle 2 n\rangle^{2} \prod_{l=3}^{n-1}\langle l n\rangle},
\end{aligned}
$$

where all legs besides $a$ and $b$ are of positive helicity, and $K_{2 \ldots(l-1)}^{\mu} \equiv \sum_{i=2}^{l-1} k_{i}^{\mu}$, and $\mathcal{P}(3,4, \ldots, n-1)$ indicates the summation over all permutations of the labels $3,4, \ldots, n-1$. (The form here is slightly rearranged compared to the one in ref. [33].) This expression has been checked numerically through 11-points against the Kawai, Lewellen and Tye relations [33], as well as those derived from on-shell recursion [31]. Although the BGK formula 


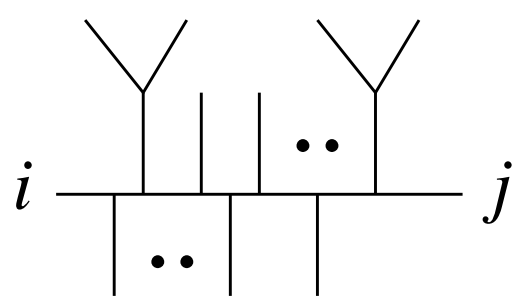

FIG. 1: A gravity Feynman diagram. Individual diagrams display bad behavior as $z \rightarrow \infty$.

has been confirmed only through 11 points, the fact that it has the correct properties as any momentum becomes soft, makes it extremely likely to be correct to all $n$. Although not manifestly so, this formula is fully crossing symmetric under an interchange of any pairs of legs, after dividing by $\langle a b\rangle^{8}$. The $\overline{\mathrm{MHV}}$ graviton amplitudes, which have two positive helicity graviton legs and the rest negative, are obtained simply by swapping angle with square brackets.

The MHV tree amplitudes satisfy simple supersymmetry Ward identities [38], allowing us to replace two of the graviton legs with any pair of particles of lesser spin,

$$
M_{n}^{\text {tree }}\left(1^{-h}, 2^{-}, 3^{+}, \ldots, n^{h}\right)=\left(\frac{\langle 2 n\rangle}{\langle 1 n\rangle}\right)^{2 h-4} M_{n}^{\text {tree }}\left(1^{-}, 2^{-}, 3^{+}, \ldots, n^{+}\right),
$$

where $a^{h}$ represents particle $a$ carrying helicity $h$, while on the right hand side of this identity, we have a pure graviton tree amplitude. For gravitons, $h$ takes on the values of \pm 2 . Similarly, for gravitinos, it takes on the values $\pm 3 / 2$, and so forth. (However, for simplicity of notation, in general, for graviton amplitudes we keep only the \pm label on each graviton leg.) At tree level this identity holds even in non-supersymmetric theories, but at loop level they hold only in supersymmetric theories. In the unitarity cuts, this identity is rather useful, giving us a simple means of separating the cancellations due to supersymmetry from those that happen with no supersymmetry. A similar identity holds for the $\overline{\mathrm{MHV}}$ amplitudes, except that angle brackets are replaced with square ones.

\section{B. Cancellations in tree amplitudes}

One clue pointing to improved high energy behavior of gravity scattering amplitudes comes from considerations of on-shell recursion relations [29, 30] for gravity amplitudes [31, 32]. These recursion relations are constructed by deforming the amplitude via a complex 
shift of momenta. For example, we may shift two of the momenta, say those of legs $i$ and $j$,

$$
k_{i}^{\mu} \rightarrow k_{i}^{\mu}(z)=k_{i}^{\mu}-\frac{z}{2}\left\langle i^{-}\left|\gamma^{\mu}\right| j^{-}\right\rangle, \quad k_{j}^{\mu} \rightarrow k_{j}^{\mu}(z)=k_{j}^{\mu}+\frac{z}{2}\left\langle i^{-}\left|\gamma^{\mu}\right| j^{-}\right\rangle,
$$

where $z$ is a complex parameter. In terms of spinor variables, the deformation (2.12) is equivalent to a shift of $\tilde{\lambda}_{i}$ and $\lambda_{j}$,

$$
\tilde{\lambda}_{i} \rightarrow \tilde{\lambda}_{i}-z \tilde{\lambda}_{j}, \quad \lambda_{j} \rightarrow \lambda_{j}+z \lambda_{i}
$$

We will refer to this as an "[i,j> shift". The above shift maintains both momentum conservation and leaves legs $i$ and $j$ on shell, deforming the amplitude $M_{n}^{\text {tree }}(z)$ to become $z$ dependent. As described in refs. [30], if a shifted tree amplitude vanishes for large $z$, it can be written as a sum over simple poles in z, giving rise to an on-shell recursion relation.

Taking $z$ in eq. (2.12) large, corresponds to taking the momenta $k_{i}(z)$ and $k_{j}(z)$ large in a complex direction, which may be interpreted as a particular high-energy limit. As discussed in refs. [31, 32], for the case of gravity, it is not at all obvious from Feynman diagrams that the shifted amplitude, $M_{n}^{\text {tree }}(z)$, will in fact vanish for large $z$. Consider the covariant single gravity Feynman diagram displayed in fig. 1, assuming we have $m$ vertices and thus $m-1$ propagators along the line connecting $\operatorname{legs} i$ and $j$. Each of these vertices scale as $z^{2}$ since two shift momenta appear at each vertex. The shifted propagators scale as $1 / z$, as can be easily checked using the fact that $\left\langle i^{-}\left|\gamma^{\mu}\right| j^{-}\right\rangle$is null. If the shifted legs $i$ and $j$ are of negative and positive helicity respectively, the polarization tensors for these legs give factors of $1 / z^{2}$ each, as can be seen by applying the shift (2.13) to eq. (2.4). Combining the various factors of $z$ gives us an overall scaling for the diagram of $z^{m-3}$ which for $m \geq 3$ is badly behaved as $z \rightarrow \infty$. In an $n$-point amplitude Feynman diagrams may have up to $m=n-2$ vertices, so the worst behaved diagrams scale as $z^{n-5}$ at large $z$. This may be contrasted to the behavior of Yang-Mills Feynman diagrams. In this case, there is at most one momentum at each vertex, leading to a large $z$ scaling in any diagram no worse than $1 / z$, after incorporating the behavior of the polarization vectors (2.3) of legs $i$ and $j$. Thus, gravity would appear to be much worse behaved at large $z$ than gauge theory, in line with the standard statements that gravity is badly behaved in the ultraviolet. For $M_{n}^{\text {tree }}(z)$ to vanish as $z \rightarrow \infty$ there need to be hidden cancellations not apparent within individual Feynman diagrams.

Indeed this is the case: various studies of on-shell recursion relations support this [31] and, more recently, Benincasa, Boucher-Veronneau and Cachazo [32] have given a general 


\begin{tabular}{|l|c|c|c|c|}
\hline \hline helicity of shifted legs & {$[-,+\rangle$} & {$[+,-\rangle$} & {$[+,+\rangle$} & {$[-,-\rangle$} \\
\hline large $z$ scaling & $z^{-2}$ & $z^{6}$ & $z^{-2}$ & $z^{-2}$ \\
\hline \hline
\end{tabular}

TABLE I: The leading scaling in $z$ of $n$-graviton amplitudes under the shift in eq. (2.12). The "+" and "-" labels refer to the helicities of the shifted legs.

proof for the vanishing of $n$-graviton amplitudes under a $[-,+\rangle$ shift, where the "-" and "+" labels refer to the helicities of the shifted legs in eq. (2.12). In particular, for MHV amplitudes, supersymmetry Ward identities give the scaling of MHV graviton amplitudes for generic shifts which we have collected in table I. This pattern has been conjectured to hold for any $n$-graviton tree amplitude [9]. By following similar reasoning as in ref. [32], for the $[-,+\rangle$ shift, we have confirmed that under a $[+,-\rangle$ shift $n$-graviton amplitudes behave as $z^{6}$ for any helicity configuration. We have also confirmed that table \is correct for all graviton helicity configurations through at least ten points, by numerically evaluating complex deformations of amplitudes constructed via on-shell recursion. The cases of $[-,-\rangle$ and $[+,+\rangle$ shift remain to be proven for $n>10$.

For MHV amplitudes, the BGK form (2.10) (or eq. (2.9) for three or four points) provides a rather simple means to confirm the pattern in table I. Under a $[1, n\rangle$ shift, ignoring the overall $\langle a b\rangle^{8}$, each term in the sum in eq. (2.10) behaves as $1 / z^{2}$ as $z \rightarrow \infty$ because $\langle 1 n\rangle$ is unshifted, $\langle 2 n\rangle \rightarrow\langle 2 n\rangle+z\langle 21\rangle$, and there is a cancellation of $z^{n-3}$ between the products in the numerator and denominator. The pattern in table I then follows, depending on whether the negative helicity legs $a$ or $b$, in eq. (2.10), correspond to legs 1 and $n$. Note that had we chosen to shift other legs, the large $z$ behavior would not be manifest. Indeed, each term would appear to have a worse behavior. However, in the sum, non-trivial cancellations between terms restore the scaling given in table I.

Interestingly, these cancellations provide some useful insight into the ultraviolet properties of gravity. The poor ultraviolet properties of gravity are usually ascribed to its two-derivative coupling, as well as the appearance of an infinite number of contact terms. The very existence of on-shell recursion relations calls into question these standard arguments. In particular, the better than expected behavior, under large $z$ deformations, suggests an improved high energy behavior, since this corresponds to a limit where momenta are becoming large in 
certain complex directions. Very remarkably, on-shell recursion relations allow us to obtain all tree amplitudes in gravity theories starting solely from the three-point vertices. The higher-point vertices that occur with conventional formulations are unnecessary. This is undoubtedly tied to the earlier realization that the higher-point vertices of gravity follow from principles of gauge and Lorentz covariance, without providing any additional dynamical information to the scattering amplitudes [39].

\section{ONE-LOOP GRAVITY AMPLITUDES AND POWER COUNTING}

Generalized unitarity allows us to use the tree-level amplitudes, described in the previous section, directly to obtain properties of loop-level amplitudes [25]. We use four-dimensional amplitudes in the cuts, allowing us to apply powerful spinor methods [35, 36]. Thanks to new developments in evaluating integrals at one loop [9, 20 23], and in particular the formalism of ref. [24], we will be able to translate straightforwardly from power counting in cuts to power counting in Feynman integrals.

\section{A. Bubble-triangle cancellations}

When starting from Feynman diagrams, a one-loop n-point amplitude is composed of a sum over loop integrals with up to $n$ propagators carrying loop momentum. Consider the generic case of an $m$-gon integral with $m$ such propagators,

$$
I_{m}=\int \frac{d^{D} l}{(2 \pi)^{D}} \frac{P_{m}(l)}{l^{2}\left(l-K_{1}\right)^{2}\left(l-K_{1}-K_{2}\right)^{2} \cdots\left(l-\sum_{j=1}^{m-1} K_{j}\right)^{2}},
$$

where the $K_{i}$ are sums over external momenta and $P_{m}(l)$ is a numerator polynomial in the loop momentum $l$. Because the integrals can contain infrared and ultraviolet divergences, we use dimensional regularization [40], analytically continuing the loop momentum integral to $D=4-2 \epsilon$ dimensions. (For the supersymmetric case we use the four-dimensional helicity scheme variant [41], which is a relative of dimensional reduction [42].)

Given an $m$-gon integral with $p$ powers of loop momentum in the numerator and massless propagators there is a standard procedure - known as Brown-Feynman or PassarinoVeltman reduction — which reduces the integral to a basis set of scalar box, triangle and bubble integrals, with no powers of loop momentum in their numerators [10, 11]. The essential trick is to trade one power of loop momentum in the numerator for a difference of 


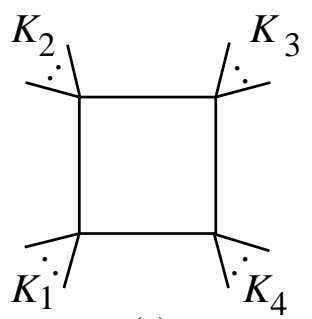

(a)

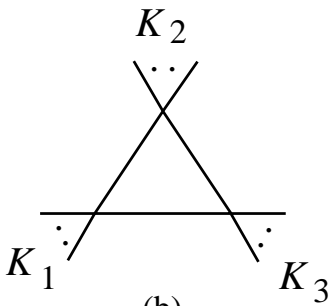

(b)

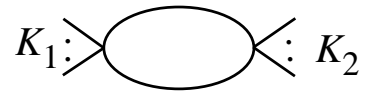

(c)

FIG. 2: The different types of box, bubble and triangle integrals are characterized by the momenta $K_{i}$ at each corner. The $K_{i}$ are sums of momenta of external particles. Non-trivial cancellations of numerator loop momentum occur within a given integral type.

inverse propagators. For example, if $1 / l^{2}$ and $1 /\left(l-k_{1}\right)^{2}$ are two propagators and $k_{1}^{2}=0$, we can rewrite,

$$
2 l \cdot k_{1}=l^{2}-\left(l-k_{1}\right)^{2}
$$

By canceling these against propagators we obtain a difference of integrals with one less propagator and one less power of loop momentum in their numerators. If instead of $2 l \cdot k_{1}$ the numerator factor was already an inverse propagator, say $l^{2}$, we could cancel two powers of loop momentum. Generically, both types of terms will appear, with the former leading to a worsening of the power counting in the reduced integrals. Although this reduction procedure cannot alter the overall ultraviolet behavior of the gravity amplitude, it does alter the power counting of individual integrals. (Cancellations between integrals restore the proper overall behavior.)

In gauge theories, in Feynman gauge, one-loop $m$-gon integrals carry up to $m$ powers of loop momentum in the numerator, due to the one-derivative coupling, and $2 m$ powers in the denominator from the propagators. Thus with increasing number of legs the ultraviolet behavior of $m$-gons gets softer in the ultraviolet. One step of the integral reductions trades one power of loop momentum in the numerator for two powers in the denominator giving $(m-1)$-gon integrals with no worse than $(m-1)$ powers of loop momentum in the numerators and $2(m-1)$ powers in the denominators from the propagators. Carrying out the chain of reduction down to the triangle level, we encounter triangle integrals with no more than three powers of loop momentum in their numerators. Similarly, carrying out the reduction down to the bubble integrals, we encounter no more than two powers of loop momentum in the numerator. This matches the power counting for the Feynman diagrams directly containing bubble and triangle loop integrals. 
In contrast, gravity $m$-gon integrals may carry up to $2 m$ powers of loop momentum in their numerators, given the two-derivative couplings of the theory, and $2 m$ powers in the denominator, since propagators are quadratic in the loop momentum. Since the power of loop momentum from vertices matches the power from the propagators, the leading ultraviolet behavior of $m$-gon integrals is independent of $m$. Starting from $P_{m}(l)$ with degree $2 m$, in carrying out the chain of integral reductions, we obtain $(m-r)$-point integrals with numerator polynomials of degree $2 m-r$ (where $r>m-2$ ). In particular, in an $n$-point gravity amplitude, under integral reductions, starting from an $n$-gon integral in the amplitude, we would obtain triangle integrals with numerator polynomials $P_{3}(l)$ of degree $n+3$ and bubble integrals with numerator polynomials $P_{2}(l)$ of degree $n+2$. That is, the triangle and bubble integrals encountered in the chain of integral reductions would appear to have a worse power count than their parent $m$-gon integrals.

The central observation of the present article is that non-trivial cancellations lead to a very different pattern for the power counting of individual integrals. We shall find that the maximum degree is six in the triangle case and four in the bubble case, i.e.

$$
P_{2}(l) \sim l^{4}, \quad P_{3}(l) \sim l^{6},
$$

independent of the number of external legs.

To investigate the power counting at the triangle level, our approach will be equivalent to reducing all $(m>3)$-point integrals to scalar box integrals and tensor triangle integrals, with no further integral reductions, as any further reductions would lower the degrees of the triangle numerator polynomials $P_{3}(l)$. Similarly, to perform the power counting at the bubble level, we will effectively reduce all $m>2$ integrals to scalar boxes, scalar triangles, and tensor bubble integrals, again with no further integral reductions that would lower the degree of the bubble numerator polynomials $P_{2}(l)$. This will allow us to demonstrate the existence of non-trivial cancellations in one-loop gravity amplitudes. We focus in our analysis on the tensor bubble and triangle integrals, since this allows us to identify cancellations straightforwardly.

To obtain a complete evaluation of the amplitude one continues the reduction procedure until only known scalar integrals remain. In this way, any dimensionally regularized one-loop amplitude can be expressed as a linear combination of basis scalar integrals multiplied by 
rational coefficients [11],

$$
A_{n}^{1 \text {-loop }}=\sum_{j} a_{j} I_{1}^{j}+\sum_{j} b_{j} I_{2}^{j}+\sum_{j} c_{j} I_{3}^{j}+\sum_{j} d_{j} I_{4}^{j}+\text { finite rational }
$$

where $I_{2}^{j}, I_{3}^{j}$ and $I_{4}^{j}$ are scalar bubble, triangle and box integrals, respectively and $b_{j}, c_{j}, d_{j}$ their rational coefficients. This structure near four dimensions, where no integrals beyond box integrals appear in the basis, is a general property. In massive theories we also obtain "cactus" or one-point contributions, $I_{1}^{j}$, but with dimensional regularization these are set to zero for massless particles, as in the cases discussed in this paper. An unwanted side effect of carrying out the complete reduction to the basis of scalar integrals, is that the power counting becomes more obscure, due to the way that dimensional regularization sets power divergences to zero. For this reason, to carry out power counting, it is much more convenient to identify cancellations prior to eliminating tensor triangle and bubble integrals. ${ }^{1}$ Besides the scalar integrals, there are also finite rational terms in eq. (3.4) which arise when the $-2 \epsilon$ dimensional components of loop momentum interfere with a $1 / \epsilon$ ultraviolet singularity. We defer the study of the finite rational terms to the future.

\section{B. Relation to one-loop finiteness}

It is useful to compare the above cancellations to the well known ultraviolet cancellations underlying the one-loop finiteness [15, 43] of pure Einstein gravity. In the language of Lagrangian counterterms one-loop pure gravity is finite because all potential counterterms vanish on shell or equivalently by the equations of motion. The only three potential one-loop counterterms are: $R^{2}, R_{\mu \nu}^{2}$, and $R_{\mu \nu \sigma \rho}^{2}$, where $R$ and $R_{\mu \nu}$ are the Ricci scalar and tensor, while $R_{\mu \nu \sigma \rho}$ is the Riemann tensor. The first two of these potential counterterms can be removed by field redefinitions and vanish on shell, while the Gauss-Bonnet theorem, in four dimensions, allows the squared Riemann tensor to be expressed as a linear combination of the other two, so it too is not a viable counterterm. This delays the divergences until two loops.

\footnotetext{
${ }^{1}$ The information on which tensor triangle or bubble integrals appear in the amplitude remains encoded in the coefficients of the basis scalar integrals via powers of the spurious poles as well as particular numerical factors.
} 
In the language of $S$-matrix elements, the ultraviolet finiteness is due to a cancellation between the coefficients of distinct bubble integrals, depending on different kinematic invariants. If the amplitude is fully reduced to the basis of scalar integrals (3.4), the ultraviolet divergences are found in bubble integrals, while the triangle and box integrals are infrared divergent, but ultraviolet finite. The explicit form of the integrals may be found, for example, in Appendix I of ref. [14]. In particular, the scalar bubble integral is,

$$
I_{2}(s)=\frac{i}{(4 \pi)^{2-\epsilon}} \frac{\Gamma(1+\epsilon) \Gamma^{2}(1-\epsilon)}{\Gamma(1-2 \epsilon)}\left(\frac{1}{\epsilon}-\ln (-s)+2\right)+\mathcal{O}(\epsilon),
$$

where $1 / \epsilon$ is an ultraviolet divergence. To see how the cancellation arises we may use the four-point amplitudes in pure gravity, as explicitly computed by Dunbar and Norridge [44]. For the pure gravity one-loop amplitude $M_{4}^{1-\text { loop }}\left(1^{-}, 2^{-}, 3^{+}, 4^{+}\right)$the bubble integrals enter in the combination,

$$
I_{2}\left(s_{14}\right)-I_{2}\left(s_{13}\right) \sim \ln \left(s_{14} / s_{13}\right),
$$

and the ultraviolet divergences cancel between the two integrals. If matter is added, the theory will no longer be one-loop finite [15, 45]. Indeed, the four-scalar amplitude, for example, diverges since the bubble integral divergences no longer cancel [44].

In contrast to the above cancellations between integrals, our study of cancellations concerns triangle and bubble integral functions within the given class specified by the external momenta at each corner, as displayed in fig. 2. We shall find that the power counting within the individual classes is better than naively expected and anticipate that this will have important consequences at higher loops, in much the same way as the no-triangle hypothesis constrains the higher-loop ultraviolet behavior in $\mathcal{N}=8$ supergravity via unitarity [2].

\section{Large momentum scaling in integrals}

First consider box integrals. The coefficients of these integrals are most easily determined from quadruple cuts [20], as shown in fig. 3(a). If we replace the four Feynman propagators by on-shell delta functions we obtain an integral of the form, ${ }^{2}$

$$
\int d^{4} l \delta\left(l^{2}\right) \delta\left(\left(l-K_{1}\right)^{2}\right) \delta\left(\left(l-K_{1}-K_{2}\right)^{2}\right) \delta\left(\left(l+K_{4}\right)^{2}\right) M_{(1)}^{\text {tree }} M_{(2)}^{\text {tree }} M_{(3)}^{\text {tree }} M_{(4)}^{\text {tree }}
$$

\footnotetext{
${ }^{2}$ Since these integrals are in general infrared divergent one need to replace $d^{4} l$ with $d^{D} l$, but this turns out to have no effect on determining the integral coefficients for $D \rightarrow 4$; this difference, however, does affect the finite rational remainder in eq. (3.4) 14].
} 


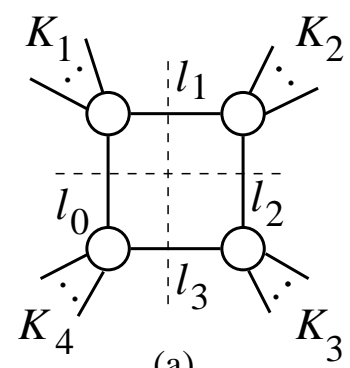

(a)

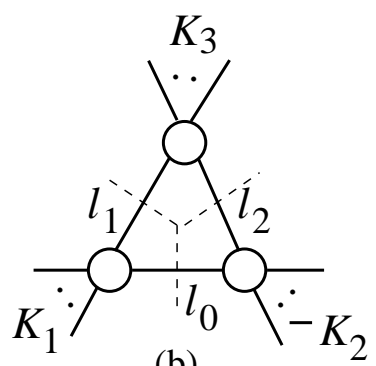

(b)

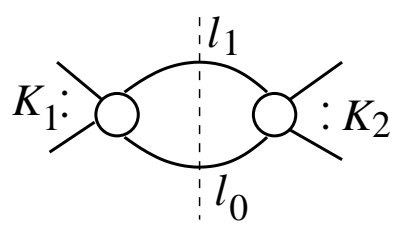

(c)

FIG. 3: The (a) quadruple, (b) triple and (c) ordinary double cut. (The minus sign in the definition of $K_{2}$ in the triple cut follows the conventions of ref. [24].) We take $l_{0} \equiv l$.

where the $M_{(i)}^{\text {tree }}$ correspond to the four tree amplitudes sitting at the corners of the box in fig. 3(a). The quadruple cut conditions freeze the loop integral in four dimensions, since there are four on-shell conditions,

$$
l^{2}=0, \quad\left(l-K_{1}\right)^{2}=0, \quad\left(l-K_{1}-K_{2}\right)^{2}=0, \quad\left(l+K_{4}\right)^{2}=0,
$$

allowing us to solve for the loop momentum directly in terms of the external momenta. (Further details and examples of calculations with quadruple cuts may be found in refs. [20, 37].) The coefficient in front of the integral is then simply given by substituting the solution for $l$ into the product of tree amplitudes. Although it is an efficient means of obtaining the rational coefficients of all basis box integrals in eq. (3.4), this has an unwanted side effect: once the quadruple cut conditions are imposed and the solved loop momentum inserted in, we can no longer perform power counting as the loop momentum has disappeared from the numerator polynomials. Similar considerations also prevent us from using generalized cuts to power count straightforwardly the $m$-gon integrals with $m>4$.

We therefore turn to triangle and bubble functions. These functions may be determined from triple and double cuts, as shown in fig. $3(\mathrm{a})$ and (b). With these cuts, there remain unfixed degrees of freedom in the loop integrals, allowing us to count powers of the loop momentum, using the parameters describing these.

Consider then the triangle integrals and the triple cuts which determine them. In this case, there are three cut conditions but four components of loop momentum, leaving one unconstrained degree of freedom. Performing a triple cut as shown in figure 3(b), allows us to isolate the triangle integral specified by the external legs at each corner. The triple cut 
is of the form,

$$
\int d^{4} l \delta\left(l^{2}\right) \delta\left(\left(l-K_{1}\right)^{2}\right) \delta\left(\left(l-K_{2}\right)^{2}\right) M_{(1)}^{\text {tree }} M_{(2)}^{\text {tree }} M_{(3)}^{\text {tree }}
$$

Following the construction of ref. [24], we parameterize the cut loop momenta in terms of the single unconstrained parameter $t$,

$$
l_{i}^{\mu}=\alpha_{i 2} K_{1}^{b, \mu}+\alpha_{i 1} K_{2}^{b, \mu}+\frac{t}{2}\left\langle K_{1}^{b,-}\left|\gamma^{\mu}\right| K_{2}^{b,-}\right\rangle+\frac{\alpha_{i 1} \alpha_{i 2}}{2 t}\left\langle K_{2}^{b,-}\left|\gamma^{\mu}\right| K_{1}^{b,-}\right\rangle
$$

where $i=0,1,2$ corresponds to the three cut lines of the triangle as shown in fig. 3(b), with $l_{0} \equiv l$. The basis momenta are,

$$
K_{1}^{b, \mu}=\frac{K_{1}^{\mu}-\left(S_{1} / \gamma\right) K_{2}^{\mu}}{1-\left(S_{1} S_{2} / \gamma^{2}\right)}, \quad K_{2}^{b, \mu}=\frac{K_{2}^{\mu}-\left(S_{2} / \gamma\right) K_{1}^{\mu}}{1-\left(S_{1} S_{2} / \gamma^{2}\right)},
$$

$S_{1}=K_{1}^{2}, S_{2}=K_{2}^{2}$ and $\gamma$ has two solutions given by,

$$
\gamma_{ \pm}=\left(K_{1} \cdot K_{2}\right) \pm \sqrt{\Delta}, \quad \Delta=\left(K_{1} \cdot K_{2}\right)^{2}-K_{1}^{2} K_{2}^{2}
$$

The $\alpha_{i j}$ parameters are functions of the $K_{i}$ whose explicit form may be found in Appendix A of ref. [24]. This parameterization is equivalent to the one used earlier in ref. [22].

With this momentum parametrization, the spinors depending on the loop momenta are,

$$
\begin{aligned}
& \left\langle l_{i}^{-}\right|=t\left\langle K_{1}^{b,-}\right|+\alpha_{i 1}\left\langle K_{2}^{b,-}\right|, \\
& \left\langle l_{i}^{+}\right|=\frac{\alpha_{i 2}}{t}\left\langle K_{1}^{b,+}\right|+\left\langle K_{2}^{b,+}\right|,
\end{aligned}
$$

so that inner products involving these are given by,

$$
\begin{array}{rlrl}
\left\langle l_{i} a\right\rangle & =t\left\langle K_{1}^{b} a\right\rangle+\alpha_{i 1}\left\langle K_{2}^{b} a\right\rangle, & {\left[l_{i} a\right]} & =\frac{\alpha_{i 2}}{t}\left[K_{1}^{b} a\right]+\left[K_{2}^{b} a\right], \\
\left\langle l_{0} l_{1}\right\rangle=-t \frac{S_{1}}{\gamma}\left\langle K_{1}^{b} K_{2}^{b}\right\rangle, & {\left[l_{0} l_{1}\right]=-\frac{1}{t}\left[K_{2}^{b} K_{1}^{b}\right],} \\
\left\langle l_{0} l_{2}\right\rangle=-t\left\langle K_{1}^{b} K_{2}^{b}\right\rangle, & {\left[l_{0} l_{2}\right]=-\frac{1}{t} \frac{S_{2}}{\gamma}\left[K_{2}^{b} K_{1}^{b}\right],} \\
\left\langle l_{1} l_{2}\right\rangle=-t\left(1-\frac{S_{1}}{\gamma}\right)\left\langle K_{1}^{b} K_{2}^{b}\right\rangle, & {\left[l_{1} l_{2}\right]=\frac{1}{t}\left(1-\frac{S_{2}}{\gamma}\right)\left[K_{2}^{b} K_{1}^{b}\right],}
\end{array}
$$

where each has a simple scaling in $t$. There is an arbitrariness in the scaling in the spinors since one can move powers of $t$ between the angle and square brackets, leaving eq. (3.10) the same. Here we use the same choice as ref. [24]. For unitarity cuts this arbitrariness cancels because the opposite helicity spinors carrying the cut loop momenta can always be paired up. 
In ref. [24] these formulæ were used to develop an efficient method for extracting the coefficients of the scalar triangle functions in the basis of integrals (3.4). Instead of evaluating integral coefficients, here we use this approach to perform power counting in each class of triangle and bubble integral functions, automatically accounting for all contributions of integral reductions of higher-point integrals. Using the cut expressions it is then a simple matter to determine the maximum numbers of loop momenta which occur in the numerators. This is done simply by making the replacements of eqs. (3.10) and (3.14) into the product of three tree amplitudes in eq. (3.9) and then determining the maximum power of $t$ in the limit that $t$ becomes large. From eq. (3.10), each power of $t$ corresponds to a power of $l$ in the numerator. This gives us the maximum tensor triangle integral that can occur had we carried out a Passarino-Veltman integral reduction on the one-loop Feynman diagrams down to triangles.

Any terms that scale as $t^{-m}$ with $m>0$ as $t \rightarrow \infty$ in the cut will not contribute to the triangle integrals. For example, a scalar box integral present in the triple cut will scale as $1 / t$ and will drop out in the large $t$ limit. If the entire triple cut scales as an inverse power of $t$, then there is no triangle contribution at all.

Next consider bubble integrals. The behavior of the bubble integrals can be obtained from the two-particle cuts, as shown in figure 3(c). Following ref. [24], the two-particle cuts leave two unconstrained parameters, which we label $y$ and $t$, allowing the cut loop momentum to be parameterized as,

$$
l_{0}^{\mu}=y K_{1}^{b, \mu}+\frac{S_{1}}{\gamma}(1-y) \chi^{\mu}+\frac{t}{2}\left\langle K_{1}^{b,-}\left|\gamma^{\mu}\right| \chi^{-}\right\rangle+\frac{S_{1}}{2 \gamma} \frac{y}{t}(1-y)\left\langle\chi^{-}\left|\gamma^{\mu}\right| K_{1}^{b,-}\right\rangle,
$$

where $l_{0} \equiv l$. Here $\chi$ is an arbitrary null momentum and

$$
K_{1}^{b, \mu}=K_{1}^{\mu}-\frac{S_{1}}{\gamma} \chi^{\mu}
$$

where $\gamma_{ \pm}=\left\langle\chi^{ \pm}\left|K_{1}\right| \chi^{ \pm}\right\rangle$.

The spinors depending on the loop momentum are,

$$
\begin{array}{ll}
\left\langle l_{0}^{-}\right|=t\left\langle K_{1}^{b,-}\right|+(1-y) \frac{S_{1}}{\gamma}\left\langle\chi^{-}\right|, & \left\langle l_{0}^{+}\right|=\frac{y}{t}\left\langle K_{1}^{b,+}\right|+\left\langle\chi^{+}\right|, \\
\left\langle l_{1}^{-}\right|=\left\langle K_{1}^{b,-}\right|-\frac{y}{t} \frac{S_{1}}{\gamma}\left\langle\chi^{-}\right|, & \left\langle l_{1}^{+}\right|=(y-1)\left\langle K_{1}^{b,+}\right|+t\left\langle\chi^{+}\right|,
\end{array}
$$

so that the inner products involving these are,

$$
\left\langle l_{0} a\right\rangle=t\left\langle K_{1}^{b} a\right\rangle+(1-y) \frac{S_{1}}{\gamma}\langle\chi a\rangle, \quad\left[l_{0} a\right]=\frac{y}{t}\left[K_{1}^{b} a\right]+[\chi a],
$$




$$
\begin{array}{rlrl}
\left\langle l_{1} a\right\rangle & =\left\langle K_{1}^{b} a\right\rangle-\frac{y}{t} \frac{S_{1}}{\gamma}\langle\chi a\rangle, & & {\left[l_{1} a\right]=(y-1)\left[K_{1}^{b} a\right]+t[\chi a],} \\
\left\langle l_{0} l_{1}\right\rangle=\frac{S_{1}}{\gamma}\left\langle K_{1}^{b} \chi\right\rangle, & {\left[l_{0} l_{1}\right]=\left[K_{1}^{b} \chi\right] .}
\end{array}
$$

In ref. [24] these were used to develop a method to extract the value of any bubble integral coefficient. Here we use it to determine the maximum number of powers of loop momenta that can occur in any bubble integral, simply by taking $y$ large and determining the maximum power of $y^{2}$. From eq. (3.15), we see that in the large $y$ limit each power of $y^{2} / t$ corresponds to an additional power $l$ that can occur in the numerator of the bubble integrals. In the formalism of ref. [24] the coefficients of bubble integrals also receive contributions from triplecut terms. However, the contributions of such terms do not alter our conclusions on the general scaling behavior of the bubble terms. Therefore, as we can always infer the overall scaling behavior of the bubble terms purely from the contributions of the two-particle cut terms, we do not need to discuss these terms further. If a two-particle cut scales as $\left(y^{2} / t\right)^{-m}$, with $m>0$, then there is no bubble contribution at all. In a similar manner to that of the triple-cut case there is an arbitrariness in the overall scaling of these spinor inner products which cancels in the cuts.

\section{POWER COUNTING OF TRIANGLE AND BUBBLE INTEGRALS}

In this section, we apply the formalism described in the previous section to perform power counting on bubble and triangle integrals in one-loop pure gravity amplitudes. First we work through some examples at six points, comparing to the earlier results obtained in $\mathcal{N}=8$

supergravity [6, 9]. Similarly we consider the other $\mathcal{N}$-extended supergravities and observe that theories with $\mathcal{N} \geq 5$ should be "cut-constructible", if the observed cancellations are universal. We then present results for an arbitrary number of legs but for limited classes of contributions. We also numerically analyze the power counting for all helicity configurations up to ten points in pure gravity. Some further results can be found in section $\mathrm{V}$, where we outline a proof of the scaling for the case when the two cut lines of each tree amplitude appearing in the cuts are of opposite helicity. 


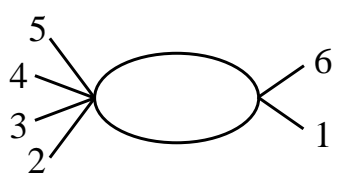

(a)

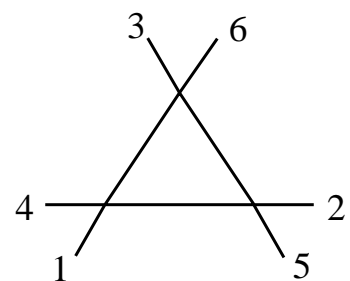

(b)

FIG. 4: Example bubble and triangle integrals appearing in the six-point amplitude.

\section{A. A six-point bubble example}

As an instructive example, consider the six-point one-loop amplitude $M_{6}^{1-\text { loop }}\left(1^{-}, 2^{-}, 3^{+}, 4^{+}, 5^{+}, 6^{+}\right)$and the power counting of the bubble integral shown in fig. 4(a). Using the Passarino-Veltman reduction, the worst behaved contribution to the bubble integral comes from the hexagon Feynman diagram displayed in fig. 5. Because of the two-derivative coupling, the hexagon Feynman diagram has 12 powers of loop momentum in the numerator. Since each step of the reduction eliminates one power of loop momentum in the numerator, as well as one propagator, after four steps we obtain bubble integrals with 8 powers loop momentum in their numerators, prior to accounting for any cancellations with other hexagon diagrams.

To obtain the maximum power of loop momentum in the bubble integral, including feed down from higher-point integrals, we determine the scaling of the two-particle cut in fig. 33(c) with $K_{1}=k_{2}+k_{3}+k_{4}+k_{5}$ and $K_{2}=k_{1}+k_{6}$, selecting the desired integral. In this case,

FIG. 5: An example of a Feynman diagram giving the worst behaved contribution to the bubble integral in fig. 4(a). Under a Passarino-Veltman reduction a hexagon integral in gravity gives bubble integrals with up to eight powers of loop momentum in the numerator. The dashed line represents the channel used to evaluate the contribution of this diagram to the bubble integral in fig. 4(a) via the unitarity cuts.

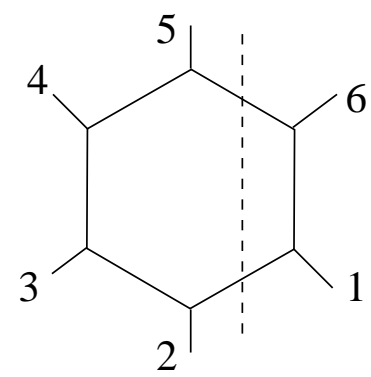


only two helicity configurations of the cut legs contribute - for the other configuration one of the tree amplitudes in the cut vanishes. Thus, the product of tree amplitudes in the cut is,

$$
\begin{aligned}
C_{61}^{\text {gravity }}= & M_{4}^{\text {tree }}\left(l_{0}^{-}, 6^{+}, 1^{-},-l_{1}^{+}\right) \times M_{6}\left(-l_{0}^{+}, 2^{-}, 3^{+}, 4^{+}, 5^{+}, l_{1}^{-}\right) \\
& +M_{4}^{\text {tree }}\left(l_{0}^{+}, 6^{+}, 1^{-},-l_{1}^{-}\right) \times M_{6}\left(-l_{0}^{-}, 2^{-}, 3^{+}, 4^{+}, 5^{+}, l_{1}^{+}\right) .
\end{aligned}
$$

First consider the behavior of the individual Feynman diagrams that compose the tree amplitudes in the cut. With the cut conditions used to determine the integrals, the large $y$ scaling is similar to that of Feynman diagrams under large $z$ scaling, described in section II, From eq. (3.15), each power of loop momentum in the numerator generically counts as one power of $y^{2}$. Since $l^{2}=0$, each propagator, $1 /\left(l-K_{i}\right)^{2} \sim 1 /\left(2 l \cdot K_{i}\right)$, counts as a power of $1 / y^{2}$. The worst behaved contribution then comes from diagrams with the maximal number of propagators and vertices on each side of the cut, an example of which is the hexagon shown in fig. 5. Diagrams with higher-point vertices or with trees attached to the loop are subdominant in the scaling. Since at six points there are a maximum of six vertices and four propagators (not counting the cut ones) this gives us a large $y$ scaling of $\left(y^{2}\right)^{12} /\left(y^{2}\right)^{4}=\left(y^{2}\right)^{8}$. (The product of polarization tensors of the cut legs have a canceling scaling in $y$.) This corresponds to a bubble integral with up to eight powers of the loop momentum $l^{\mu}$ in the numerator, matching the above counting from Passarino-Veltman integral reductions. Of course, neither of these power counts account for any cancellation between the diagrams. In order to see any such cancellations we need to use the explicit form of the tree amplitudes in the cuts.

As a warm-up prior to evaluating the gravity cut (4.1), it is useful to consider the cut analysis in Yang-Mills theory. The corresponding unitarity cut with the external legs color ordered is given by,

$$
\begin{aligned}
C_{61}^{\mathrm{YM}}=A_{4}^{\text {tree }} & \left(l_{0}^{-}, 6^{+}, 1^{-},-l_{1}^{+}\right) \times A_{6}^{\text {tree }}\left(-l_{0}^{+}, 2^{-}, 3^{+}, 4^{+}, 5^{+}, l_{1}^{-}\right) \\
& +A_{4}^{\text {tree }}\left(l_{0}^{+}, 6^{+}, 1^{-},-l_{1}^{-}\right) \times A_{6}^{\text {tree }}\left(-l_{0}^{-}, 2^{-}, 3^{+}, 4^{+}, 5^{+}, l_{1}^{+}\right) .
\end{aligned}
$$

The first of these terms is given by,

$$
\frac{i\left\langle 1 l_{0}\right\rangle^{4}}{\left\langle l_{1} l_{0}\right\rangle\left\langle l_{0} 6\right\rangle\langle 61\rangle\left\langle 1 l_{1}\right\rangle} \times \frac{i\left\langle l_{1} 2\right\rangle^{4}}{\left\langle l_{1} l_{0}\right\rangle\left\langle l_{0} 2\right\rangle\langle 23\rangle\langle 34\rangle\langle 45\rangle\left\langle 5 l_{1}\right\rangle} \sim y^{2} t \times y^{2} / t^{3} \sim\left(l^{\mu}\right)^{2},
$$


where we used the explicit forms of the MHV gluon amplitudes [36, 46]. To obtain the large$y$ scaling we use eq. (3.18). Thus, we have a maximum of two powers of loop momentum in the bubble integrals from the first term. The second term in eq. (4.2) is similar,

$$
\frac{i\left\langle 1 l_{1}\right\rangle^{4}}{\left\langle l_{1} l_{0}\right\rangle\left\langle l_{0} 6\right\rangle\langle 61\rangle\left\langle 1 l_{1}\right\rangle} \times \frac{i\left\langle l_{0} 2\right\rangle^{4}}{\left\langle l_{1} l_{0}\right\rangle\left\langle l_{0} 2\right\rangle\langle 23\rangle\langle 34\rangle\langle 45\rangle\left\langle 5 l_{1}\right\rangle} \sim \frac{y^{2}}{t^{3}} \times y^{2} t \sim\left(l^{\mu}\right)^{2} .
$$

Thus, we reproduce the well know result that bubble integrals in gauge theory can have up to two powers of loop momentum in their numerators, including the contribution from the reduction of higher-point tensor integrals.

The KLT relations (2.6) and (2.8) give us a simple way to compare the gravity bubble integral power counting directly to one for gauge theory and to identify cancellations. Using the labels appearing in the gravity cut (4.1), we have from the KLT relations,

$$
\begin{aligned}
M_{4}^{\text {tree }}\left(l_{0}, 6,1,-l_{1}\right)= & -i s_{61} A_{4}^{\text {tree }}\left(l_{0}, 6,1,-l_{1}\right) A_{4}^{\text {tree }}\left(l_{0}, 1,6,-l_{1}\right), \\
M_{6}^{\text {tree }}\left(-l_{0}, 2,3,4,5, l_{1}\right)= & -i s_{l_{0} 2} s_{45} A_{6}^{\text {tree }}\left(-l_{0}, 2,3,4,5, l_{1}\right)\left(s_{l_{0} 3} A_{6}^{\text {tree }}\left(2,3,-l_{0}, 5,4, l_{1}\right)\right. \\
& \left.\quad+\left(s_{l_{0} 3}-s_{23}\right) A_{6}^{\text {tree }}\left(3,2,-l_{0}, 5,4, l_{1}\right)\right)+\mathcal{P}(2,3,4),
\end{aligned}
$$

where the six-point expression is obtained from eq. (2.8), by first relabeling $(1,2,3,4,5,6) \rightarrow$ $\left(5,4,3,2,-l_{0}, l_{1}\right)$, then using the invariance of the color-ordered gauge-theory tree amplitudes under cyclic permutations and reversal of leg labels.

Using these expressions it is straightforward to evaluate the large $y$ scaling in the gravity cut in eq. (4.1). For the four-point amplitudes appearing in the cut we have,

$$
\begin{aligned}
& M_{4}^{\text {tree }}\left(l_{0}^{-}, 6^{+}, 1^{-},-l_{1}^{+}\right)=-i s_{61} \frac{i\left\langle 1 l_{0}\right\rangle^{4}}{\left\langle l_{1} l_{0}\right\rangle\left\langle l_{0} 6\right\rangle\langle 61\rangle\left\langle 1 l_{1}\right\rangle} \frac{i\left\langle 1 l_{0}\right\rangle^{4}}{\left\langle l_{1} l_{0}\right\rangle\left\langle l_{0} 1\right\rangle\langle 16\rangle\left\langle 6 l_{1}\right\rangle} \sim y^{4} t^{2}, \\
& M_{4}^{\text {tree }}\left(l_{0}^{+}, 6^{+}, 1^{-},-l_{1}^{-}\right)=-i s_{61} \frac{i\left\langle 1 l_{1}\right\rangle^{4}}{\left\langle l_{1} l_{0}\right\rangle\left\langle l_{0} 6\right\rangle\langle 61\rangle\left\langle 1 l_{1}\right\rangle} \frac{i\left\langle 1 l_{1}\right\rangle^{4}}{\left\langle l_{1} l_{0}\right\rangle\left\langle l_{0} 1\right\rangle\langle 16\rangle\left\langle 6 l_{1}\right\rangle} \sim y^{4} t^{-6} .
\end{aligned}
$$

On the other side of the cut, for the six-point tree amplitudes, some terms appear to be worse behaved because of the explicit kinematic invariants carrying loop momentum appearing in the KLT expression (4.6). However, there is a non-trivial cancellation in such terms. In particular, consider the following terms in the six-point amplitude in eq. (4.6) with helicity configuration $M_{6}^{\text {tree }}\left(-l_{0}^{+}, 2^{-}, 3^{+}, 4^{+}, 5^{+}, l_{1}^{-}\right)$:

$$
\begin{aligned}
& s_{l_{0} 2} s_{l_{0} 3} A_{6}^{\text {tree }}\left(-l_{0}^{+}, 2^{-}, 3^{+}, 4^{+}, 5^{+}, l_{1}^{-}\right) \\
& \quad \times\left(A_{6}^{\text {tree }}\left(2^{-}, 3^{+},-l_{0}^{+}, 5^{+}, 4^{+}, l_{1}^{-}\right)+A_{6}^{\text {tree }}\left(3^{+}, 2^{-},-l_{0}^{+}, 5^{+}, 4^{+}, l_{1}^{-}\right)\right)
\end{aligned}
$$




$$
\begin{aligned}
& =s_{l_{0} 2} s_{l_{0} 3} \frac{i\left\langle l_{1} 2\right\rangle^{4}}{\left\langle l_{1} l_{0}\right\rangle\left\langle l_{0} 2\right\rangle\langle 23\rangle\langle 34\rangle\langle 45\rangle\left\langle 5 l_{1}\right\rangle} \frac{i\left\langle l_{1} 2\right\rangle^{4}}{\left\langle l_{0} 5\right\rangle\langle 54\rangle\left\langle 4 l_{1}\right\rangle\langle 23\rangle}\left(\frac{1}{\left\langle l_{1} 2\right\rangle\left\langle 3 l_{0}\right\rangle}-\frac{1}{\left\langle l_{1} 3\right\rangle\left\langle 2 l_{0}\right\rangle}\right) \\
& =s_{l_{0} 2} s_{l_{0} 3} \frac{i\left\langle l_{1} 2\right\rangle^{4}}{\left\langle l_{1} l_{0}\right\rangle\left\langle l_{0} 2\right\rangle\langle 23\rangle\langle 34\rangle\langle 45\rangle\left\langle 5 l_{1}\right\rangle} \frac{i\left\langle l_{1} 2\right\rangle^{4}}{\left\langle l_{0} 5\right\rangle\langle 54\rangle\left\langle 4 l_{1}\right\rangle} \frac{\left\langle l_{1} l_{0}\right\rangle}{\left\langle l_{1} 2\right\rangle\left\langle 3 l_{0}\right\rangle\left\langle l_{1} 3\right\rangle\left\langle 2 l_{0}\right\rangle} \\
& \sim y^{4} t^{-6},
\end{aligned}
$$

where we made use of the Schouten identity (2.2). Note the explicit factor of $s_{l_{0} 2} s_{l_{0} 3}$ in front of these terms, which makes the expression appear badly behaved. However, there are compensating reductions in the scaling. One reduction comes from $l_{1}$ and $l_{0}$ being non-adjacent in the color ordering of one of the Yang-Mills factors. In addition, there is a non-trivial cancellation between the terms again reducing the scaling by another power of $y^{2}$, giving the overall scaling of $y^{4}$. It is not difficult to check that all the remaining terms in eq. (4.6) scale the same way. The net effect is that the six-point tree amplitudes appearing in the cut (4.1) scale as,

$$
\begin{aligned}
& M_{6}^{\text {tree }}\left(-l_{0}^{+}, 2^{-}, 3^{+}, 4^{+}, 5^{+}, l_{1}^{-}\right) \sim y^{4} t^{-6}, \\
& M_{6}^{\text {tree }}\left(-l_{0}^{-}, 2^{-}, 3^{+}, 4^{+}, 5^{+}, l_{1}^{+}\right) \sim y^{4} t^{2} .
\end{aligned}
$$

Combining this scaling with that of eq. (4.7) gives us that the two particle cut (4.1) scales as,

$$
C_{61}^{\text {gravity }} \sim y^{8} t^{-4} \sim\left(l^{\mu}\right)^{4}
$$

We thus conclude that the maximum number of powers of loop momentum that can appear in the numerator of the gravity bubble integral shown in fig. $4(a)$ is $\left(l^{\mu}\right)^{4}$. This is significantly fewer powers than the $\left(l^{\mu}\right)^{8}$ that is found from the integral reduction of an individual hexagon gravity Feynman diagram down to bubble integrals. It is important to note the non-trivial cancellation in eq. (4.8) required to obtain this result: only after the terms are combined do we find the better scaling behavior.

At this point we may compare to the known $\mathcal{N}=8$ supergravity six-graviton MHV amplitude [6], in order to separate the cancellations due to supersymmetry from those inherent to generic gravity theories. The supergravity case is quite similar to the pure gravity case, except that we need to sum over the contributions of the super-multiplet in the loop. Thanks to the MHV supersymmetry Ward identities (2.11) this task is straightforward. In $\mathcal{N}=8$ supergravity, we must sum over the contribution of the 256 different states of the theory. These correspond to one graviton, 8 gravitinos, 28 vectors, 56 spin $1 / 2$ fermions, and 70 real 
scalars. Using the supersymmetry Ward identity (2.11) and taking the contribution of the super-multiplet into account by an overall factor $\rho_{8}$, we have,

$$
\begin{aligned}
C_{61}^{\mathcal{N}=8} & =\sum_{h \in \mathcal{N}=8 \text { multiplet }} M_{4}^{\text {tree }}\left(l_{0}^{-h}, 6^{+}, 1^{-},-l_{1}^{h}\right) \times M_{6}\left(-l_{0}^{h}, 2^{-}, 3^{+}, 4^{+}, 5^{+}, l_{1}^{-h}\right) \\
& =\rho_{8} \times M_{4}^{\text {tree }}\left(l_{0}^{-}, 6^{+}, 1^{-},-l_{1}^{+}\right) \times M_{6}\left(-l_{0}^{+}, 2^{-}, 3^{+}, 4^{+}, 5^{+}, l_{1}^{-}\right)
\end{aligned}
$$

where

$$
\begin{aligned}
\rho_{8} & =\left(1-8 x+28 x^{2}-56 x^{3}+70 x^{4}-56 x^{5}+28 x^{6}-8 x^{7}+x^{8}\right) \\
& =(1-x)^{8}, \quad x=\frac{\left\langle l_{1} 1\right\rangle\left\langle l_{0} 2\right\rangle}{\left\langle l_{0} 1\right\rangle\left\langle l_{1} 2\right\rangle} .
\end{aligned}
$$

The relative minus signs between the terms are due to the minus signs associated with fermionic loops. Applying the Schouten identity (2.2) gives,

$$
\rho_{8}=\left(\frac{\left\langle l_{1} l_{0}\right\rangle\langle 12\rangle}{\left\langle l_{0} 1\right\rangle\left\langle l_{1} 2\right\rangle}\right)^{8} \sim y^{-16} t^{8} \sim\left(l^{\mu}\right)^{-8} .
$$

The scaling of $\rho_{8}$ makes manifest the cancellations of eight powers of loop momentum $l$ due to supersymmetry. In total, the cut scales as $y^{-8} t^{4}$ implying the vanishing of the bubble coefficient which confirms the known result [6]. Of the $n-6$ powers of loop momentum that cancel to eliminate the bubble integrals in the $\mathcal{N}=8 n$-graviton amplitude, only eight are due to supersymmetry. Thus, to a large extent, the one-loop cancellations in the $\mathcal{N}=8$ theory originate from cancellations present in the non-supersymmetric pure gravity case. This is a central result of this paper.

Cases with fewer supersymmetries are similar. For example, the $\mathcal{N}=1$ gravity multiplet consisting of a graviton and gravitino in the loop, has the corresponding factor of,

$$
\rho_{1}=1-x-x^{7}+x^{8} \sim y^{-4} t^{2} \sim\left(l^{\mu}\right)^{-2}
$$

where $x$ is given in eq. (4.13). Note that there is an additional cancellation in this expression, besides the leading one, reducing the power of loop momentum by two. Similarly, for an $\mathcal{N}=2$ gravity multiplet, the factor is,

$$
\rho_{2}=1-2 x+x^{2}+x^{6}-2 x^{7}+x^{8} \sim y^{-4} t^{2} \sim\left(l^{\mu}\right)^{-2},
$$

so that the additional supersymmetry has not reduced the power count compared to the $\mathcal{N}=1$ case. We have checked that this pattern generalizes to higher supersymmetry. Depending on whether the number of supersymmetries is even or odd we have,

$$
\rho_{\mathcal{N}_{\text {even }}} \sim\left(l^{\mu}\right)^{-\mathcal{N}_{\text {even }}}, \quad \quad \rho_{\mathcal{N}_{\text {odd }}} \sim\left(l^{\mu}\right)^{-\left(\mathcal{N}_{\text {odd }}+1\right)} .
$$


It is interesting to note that, according to this pattern, theories with $\mathcal{N} \geq 3$ can have no powers of loop momentum in the numerators of bubble integrals, and therefore no additional rational terms from here [14]. ${ }^{3}$ A further interesting bound is $\mathcal{N} \geq 5$, where the above counting implies that bubble integrals will not be present in the one-loop amplitudes (3.4). In addition, there can be at most three powers of loop momentum in box numerators, which is insufficient to generate rational terms.

\section{B. A six-point triangle example}

As a second example, consider the next-to-MHV (NMHV) amplitude $M_{4}^{1-\text { loop }}\left(1^{-}, 2^{-}, 3^{-}, 4^{+}, 5^{+}, 6^{+}\right)$and the power counting of the triangle integral shown in fig. 4(b). As described in the previous section, to analyze the power counting in triangle functions, we consider triple cuts. Two helicity configurations of cut legs contribute,

$$
\begin{aligned}
C_{14,25,36}^{\text {gravity }}= & M_{4}^{\text {tree }}\left(l_{0}^{-}, 1^{-}, 4^{+},-l_{1}^{+}\right) \times M_{4}^{\text {tree }}\left(l_{1}^{-}, 2^{-}, 5^{+},-l_{2}^{+}\right) \times M_{4}^{\text {tree }}\left(l_{2}^{-}, 3^{-}, 6^{+},-l_{0}^{+}\right) \\
& +M_{4}^{\text {tree }}\left(l_{0}^{+}, 1^{-}, 4^{+},-l_{1}^{-}\right) \times M_{4}^{\text {tree }}\left(l_{1}^{+}, 2^{-}, 5^{+},-l_{2}^{-}\right) \times M_{4}^{\text {tree }}\left(l_{2}^{+}, 3^{-}, 6^{+},-l_{0}^{-}\right) .
\end{aligned}
$$

First consider the naive power counting of the individual Feynman diagrams contributing to the tree amplitudes appearing in the cut. From eq. (3.10), we see that each numerator loop momentum scales as $t$. In the denominator, any propagators in the tree amplitudes $1 /\left(l-K_{i}\right)^{2}$ scale as $1 / t$ because of the on-shell condition $l^{2}=0$. Using this scaling we thus obtain the leading behavior from the two vertices and one propagator in each of the four-point tree-amplitudes giving a total of

$$
\left(t^{2} \times t^{2} / t\right)^{3} \sim t^{9} \sim\left(l^{\mu}\right)^{9} .
$$

This corresponds to the eight powers of loop momentum we obtained in the bubble integrals starting from a hexagon Feynman diagram, because the bubble integrals require one more step in the reduction compared to triangles.

Now consider power counting in the triangle integral of fig. 4(b), while accounting for cancellations between diagrams. Using the explicit form of the tree amplitudes and spinor

\footnotetext{
${ }^{3}$ For $\mathcal{N} \leq 4$ supergravity there can be rational contributions from box diagrams, because a reduction of four powers of loop momentum still leaves four powers behind which under integral reduction gives rational terms. We thank L. Dixon and C. Boucher-Veronneau for pointing this out. This is consistent with the results of ref. [47].
} 
products collected in (3.14), for the first term in eq. (4.18) we have,

$$
M_{4}^{\text {tree }}\left(l_{0}^{-}, 1^{-}, 4^{+},-l_{1}^{+}\right)=i \frac{[41]\left\langle l_{0} 1\right\rangle^{7}}{\left\langle l_{1} l_{0}\right\rangle^{2}\langle 14\rangle\left\langle 4 l_{1}\right\rangle\left\langle l_{0} 4\right\rangle\left\langle 1 l_{1}\right\rangle} \sim t^{2} .
$$

The other factors of tree amplitudes in this term are similar, each also giving a factor of $t^{2}$. Thus, the first term in eq. (4.18) scales as $t^{6}$, indicating six powers of loop momentum: $\left(l^{\mu}\right)^{6}$. Similarly, the second term in eq. (4.18) also scales the same way, giving us an overall scaling of

$$
C_{14,25,36}^{\text {gravity }} \sim t^{6} \sim\left(l^{\mu}\right)^{6}
$$

The improved scaling is due to cancellations between Feynman diagrams in the tree amplitudes.

We may compare this to the results of ref. [9] for the $\mathcal{N}=8$ theory. As for the bubble integral case, the sum over the $\mathcal{N}=8$ multiplet in the loop can be included by a multiplicative factor,

$$
\begin{aligned}
C_{14,25,36}^{\mathcal{N}=8}= & \sum_{h \in \mathcal{N}=8 \text { states }} M_{4}^{\text {tree }}\left(l_{0}^{-h}, 1^{-}, 4^{+},-l_{1}^{h}\right) \times M_{4}^{\text {tree }}\left(l_{1}^{-h}, 2^{-}, 5^{+},-l_{2}^{h}\right) \\
& \times M_{4}^{\text {tree }}\left(l_{2}^{-h}, 3^{-}, 6^{+},-l_{0}^{h}\right) \\
= & \rho_{8} \times M_{4}^{\text {tree }}\left(l_{0}^{-}, 1^{-}, 4^{+},-l_{1}^{+}\right) \times M_{4}^{\text {tree }}\left(l_{1}^{-}, 2^{-}, 5^{+},-l_{2}^{+}\right) \times M_{4}^{\text {tree }}\left(l_{2}^{-}, 3^{-}, 6^{+},-l_{0}^{+}\right),
\end{aligned}
$$

where,

$$
\rho_{8}=(1-x)^{8}, \quad x=\frac{\left\langle l_{1} 1\right\rangle\left\langle l_{2} 2\right\rangle\left\langle l_{0} 3\right\rangle}{\left\langle l_{0} 1\right\rangle\left\langle l_{1} 2\right\rangle\left\langle l_{2} 3\right\rangle}
$$

From eq. (3.14) we have that $\left\langle l_{i} a\right\rangle \sim t\left\langle K_{1}^{b} a\right\rangle$, so that the leading $t$ behavior cancels, giving us,

$$
\rho_{8} \sim t^{-8}
$$

Thus, as in the bubble case, the $\mathcal{N}=8$ supersymmetry reduces the degree of the loop momentum polynomial by eight.

In total, combining eqs. (4.21) and (4.24) shows the triple-cut integral scales to zero, like $t^{-2}$, for $\mathcal{N}=8$ supergravity so that the triangle integral is not present in the amplitude. However, only because of the cancellations already present in pure gravity does the triangle integral coefficient vanish. The vanishing of this triangle integral coefficient in the $\mathcal{N}=8$ theory has been shown numerically in ref. [9]. The analysis here provides a simple analytic proof of the vanishing. 
As for the bubble integral discussed above, the cases with less supersymmetry are similar. Depending on whether the number of supersymmetries is odd or even, we have either $\mathcal{N}+1$ or $\mathcal{N}$ powers of canceled loop momentum, respectively.

\section{All- $n$ power counting}

We now discuss power counting in the bubble and triangle integrals for an arbitrary number of external legs. We will discuss the cancellations in the two cases, and close with a summary of the checks that we have performed.

\section{Bubble integrals}

As described above, in order to perform all- $n$ power counting for the bubble functions shown in fig. 2(c), we determine the leading power of $y^{2} / t$ under the large- $y$ scaling properties of the tree amplitudes contributing to the two-particle cuts of fig. 3(c). There are two possible helicity configurations crossing the two-particle cuts. Either the legs crossing the cuts are

of the same helicity or they are of opposite helicity. If they are of opposite helicity, then the contribution to the cuts is,

$$
\begin{aligned}
C_{2}^{(-,+)}= & M_{m+2}^{\text {tree }}\left(l_{0}^{-}, i_{1}, \ldots i_{m},-l_{1}^{+}\right) \times M_{n-m+2}^{\text {tree }}\left(l_{1}^{-}, i_{1}^{\prime}, \ldots i_{n-m}^{\prime},-l_{0}^{+}\right) \\
& +M_{m+2}^{\text {tree }}\left(l_{0}^{+}, i_{1}, \ldots i_{m},-l_{1}^{-}\right) \times M_{n-m+2}^{\text {tree }}\left(l_{1}^{+}, i_{1}^{\prime}, \ldots i_{n-m}^{\prime},-l_{0}^{-}\right),
\end{aligned}
$$

where we identify $l_{0}$ with the loop momentum $l$. If the two legs crossing the cut are of the same helicity the contribution is,

$$
\begin{aligned}
C_{2}^{(+,+)}= & M_{m+2}^{\text {tree }}\left(l_{0}^{+}, i_{1}, \ldots i_{m},-l_{1}^{+}\right) \times M_{n-m+2}^{\text {tree }}\left(l_{1}^{-}, i_{1}^{\prime}, \ldots i_{n-m}^{\prime},-l_{0}^{-}\right) \\
& +M_{m+2}^{\text {tree }}\left(l_{0}^{-}, i_{1}, \ldots i_{m},-l_{1}^{-}\right) \times M_{n-m+2}^{\text {tree }}\left(l_{1}^{+}, i_{1}^{\prime}, \ldots i_{n-m}^{\prime},-l_{0}^{+}\right) .
\end{aligned}
$$

For cases where the tree amplitudes in the cuts are either MHV or $\overline{\mathrm{MHV}}$, we can read off the scaling in $y$, by choosing leg $l_{0}$ and $l_{1}$ to correspond to legs 1 and $n$ in eqs. (2.9) and (2.10), or their parity conjugates. With this choice, the scaling properties in $y$ and $t$ from eq. (3.18) are manifest in each term. Choosing the negative helicity legs in $a$ and $b$, to match the tree amplitude appearing in the cuts, we can easily evaluate all cases, collected in table II. For more general helicities we do not have a complete proof, but we have numerically checked 


\begin{tabular}{|l|c|c|c|c|}
\hline \hline$\left(h_{l_{0}},-h_{l_{1}}\right)$ & $(-,+)$ & $(+,-)$ & $(+,+)$ & $(-,-)$ \\
\hline$y$-scaling of $M_{n}^{\text {tree }}$ & $y^{4} t^{2}$ & $y^{4} t^{-6}$ & $y^{-4} t^{2}$ & $y^{-4} t^{2}$ \\
\hline \hline
\end{tabular}

TABLE II: The large $y$ scaling of tree-amplitudes appearing in the two-particle cuts of a one-loop amplitude. The scaling depends on the helicities of the two legs carrying loop momentum, indicated in the first row. The amplitudes are evaluated using the cut kinematics in eqs. (3.15) and (3.18).

that the scaling in table II is correct for all helicity configurations up to ten points. We have also constructed an $n$-point proof for the cases where opposite helicities cross the cut, outlined in section $\mathrm{V}$.

Using table II, we can then read off the maximum scaling of the cuts (4.25) and (4.26) at large $y$,

$$
\begin{aligned}
& C_{2}^{(-,+)} \sim y^{4} t^{2} \times y^{4} t^{-6} \sim\left(l^{\mu}\right)^{4} \\
& C_{2}^{(+,+)} \sim y^{-4} t^{2} \times y^{-4} t^{2} \sim\left(l^{\mu}\right)^{-4} .
\end{aligned}
$$

Thus, for the case where opposite helicities cross the cut, up to four powers of loop momentum appear in the numerator of the bubble integral. For the case where like helicities cross the cut, the bubble contributions cancel completely, as indicated by the negative power of $y$. Both cases exhibit non-trivial cancellations compared to individual Feynman diagrams. For $n \leq 10$ external gravitons our numerical analysis directly confirms eq. (4.27) for all helicity configurations.

We may compare these results to the corresponding ones for an $\mathcal{N}$-extended supergravity. The case with identical helicities crossing the cut is the same in the two theories and there are no contributing bubble integrals. For the case of opposite helicities crossing the cut, we need to sum over the contributions of the entire super-multiplet. For the case of MHV or $\overline{\mathrm{MHV}}$ amplitudes, it is simple to carry out the sum over the multiplet, using the supersymmetry Ward identify (2.11). As for the six-point case, with opposite helicity legs crossing the cuts, the net effect from the super-multiplet sum is the additional overall factor of $\rho_{\mathcal{N}}$ given in eq. (4.17).

Specializing to $\mathcal{N}=8$, the bubble contributions all scale as $y^{-4} t^{2}$, so there are no bubble integrals present in the MHV amplitudes, as already discussed in refs. [6, 9]. As for 


\begin{tabular}{|l|c|c|c|c|}
\hline \hline$\left(h_{l_{i}},-h_{l_{i+1}}\right)$ & $(-,+)$ & $(+,-)$ & $(+,+)$ & $(-,-)$ \\
\hline$t$-scaling of $M_{n}^{\text {tree }}$ & $t^{2}$ & $t^{2}$ & $t^{-6}$ & $t^{2}$ \\
\hline \hline
\end{tabular}

TABLE III: The leading $t$-scaling of tree amplitudes appearing in the triple-cuts. The tree amplitudes are evaluated using the spinor inner products in eq. (3.14). The first row corresponds to the helicities of the cut legs carrying loop momentum.

the six-point example above, we find that the supersymmetry only reduces the number of loop momenta by eight powers when opposite helicities cross the cut, with the remaining cancellations present even in pure gravity. When like helicities cross the cut, the $\mathcal{N}=8$ cancellations are identical to those of pure gravity.

\section{Triangle integrals}

The power counting of the triangle integral of fig. 2(b) is determined by the large- $t$ scaling of the three tree amplitudes composing the triple cut of fig. 3(b). For example, the contribution, where each tree amplitude has opposite helicity cut legs, is given by,

$$
\begin{aligned}
C_{3} & =M_{m+2}^{\text {tree }}\left(l_{0}^{+}, i_{1}, \ldots i_{m},-l_{1}^{-}\right) \times M_{m^{\prime}+2}^{\text {tree }}\left(l_{1}^{+}, i_{1}^{\prime}, \ldots i_{m^{\prime}}^{\prime},-l_{2}^{-}\right) \times M_{m^{\prime \prime}+2}^{\text {tree }}\left(l_{2}^{+}, i_{1}^{\prime \prime}, \ldots i_{m^{\prime \prime}}^{\prime \prime},-l_{0}^{-}\right) \\
& +M_{m+2}^{\text {tree }}\left(l_{0}^{-}, i_{1}, \ldots i_{m},-l_{1}^{+}\right) \times M_{m^{\prime}+2}^{\text {tree }}\left(l_{1}^{-}, i_{1}^{\prime}, \ldots i_{m^{\prime}}^{\prime},-l_{2}^{+}\right) \times M_{m^{\prime \prime}+2}^{\text {tree }}\left(l_{2}^{-}, i_{1}^{\prime \prime}, \ldots i_{m^{\prime \prime}}^{\prime \prime},-l_{0}^{+}\right)
\end{aligned}
$$

where $m+m^{\prime}+m^{\prime \prime}=n$ for an $n$-point amplitude.

Again, with MHV or $\overline{\mathrm{MHV}}$ tree amplitudes appearing in the triple cuts, it is a simple matter to obtain the scaling pattern in table III from eqs. (2.9) and (2.10) and their parity conjugates, by choosing the legs carrying loop momentum to be legs 1 and $n$. For all helicity configurations, we have numerically checked that the table is correct up to ten points, using the kinematics of eqs. (3.10) and (3.14). An all-n proof for the particular helicity configurations $( \pm, \mp)$ crossing the cut will be outlined in section $\mathrm{V}$.

Given the scalings in table III we see that no product of the three tree amplitudes in a triple cut can have worse scaling than,

$$
C_{3} \sim t^{2} \times t^{2} \times t^{2} \sim\left(l^{\mu}\right)^{6}
$$

including contributions from integral reductions from higher-point integrals. Again, this is 
significantly better than the behavior obtained from an $n$-gon Feynman diagram which can generate triangles integrals with up to $n+3$ powers of loop momentum in their numerators.

For the cases where the cuts are composed of purely MHV and $\overline{M H V}$ amplitudes, we can easily compare to the results of $\mathcal{N}=8$ supergravity, by making use of the supersymmetry identity (2.11). In all cases, we obtain an overall scaling of $t^{-2} \sim\left(l^{\mu}\right)^{-2}$, including any additional cancellations from supersymmetry, which means that triangle integrals are not present. As in our previous examples, the key $n$-dependent cancellations are already present in the non-supersymmetric case.

\section{Summary of checks}

In summary our checks confirm cancellations for pure Einstein gravity within each triangle and bubble contribution. We confirm these cancellations when the tree amplitudes appearing the cuts determining the integrals are from among the following:

1. An m-point MHV amplitude.

2. An $m$-point $\overline{\mathrm{MHV}}$ amplitude.

3. Any helicity configuration with up to ten gravitons.

4. An $m$-point amplitude with loop-momentum helicities $( \pm, \mp){ }^{4}$

This demonstrates that the one-loop cancellations hold through ten points in pure gravity for all helicity configurations and argues strongly that it continues to hold beyond this. One might be concerned that the results for the MHV and $\overline{\mathrm{MHV}}$ amplitudes were derived using the BGK formula, which has been tested only up to 11 points and not beyond this. This is not a real restriction here, as the information we extract from the BGK formula is its scaling properties, which will be shown to hold for all $m$ by an independent line of reasoning in section $\mathrm{Q}$,

For cases with only MHV or $\overline{M H V}$ amplitudes in the cuts we analyzed the additional cancellations in $\mathcal{N}$-extended supergravity theories by summing over the super-multiplet. When opposite helicities cross the cut, depending on whether $\mathcal{N}$ is even or odd we have additional

\footnotetext{
${ }^{4}$ This check will be discussed below in section $\square$
} 
cancellations of $\mathcal{N}$ or $(\mathcal{N}+1)$ powers of loop momentum, respectively. In particular, for $\mathcal{N}=8$ supergravity, the no-triangle hypothesis follows from a combination of cancellations, with at most eight powers of loop momentum in the numerator canceled by supersymmetry and the remaining cancellations already present in pure gravity.

\section{RELATIONS BETWEEN DIFFERENT CANCELLATIONS}

We now discuss a proof that the one-loop cancellations described above are simply related to the tree-level large- $z$ cancellations under the shift (2.13). We also present a heuristic argument using factorization to explain the pure gravity cancellations, similar to the one used to propose the "no-triangle hypothesis" for $\mathcal{N}=8$ supergravity in ref. [7].

\section{A. Relations between tree and triangle-bubble scaling properties}

Consider a generic tree amplitude,

$$
M_{n}^{\text {tree }}\left(l_{0}^{h_{0}},-l_{1}^{h_{1}}, i_{1}, \ldots, i_{n-2}\right),
$$

where $h_{0}$ and $h_{1}$ denote the helicities - for gravitons these take on the values of \pm 2 . We now observe some simple relations between the different scalings. Suppose that after a $\left[l_{0},-l_{1}\right\rangle$ shift (2.12), and after accounting for all cancellations, we find that the tree amplitude scales in the large- $z$ limit as,

$$
\left[l_{0},-l_{1}\right\rangle: \quad M_{n}^{\text {tree }}(z) \sim z^{m}
$$

where the value of $m$ depends upon the helicities. Using table I, we see that the scaling of the flipped $\left[-l_{1}, l_{0}\right\rangle$ shift, at large $z$, is related to the above by,

$$
\left[-l_{1}, l_{0}\right\rangle: \quad M_{n}^{\text {tree }}(z) \sim z^{m+2\left(h_{1}-h_{0}\right)} .
$$

Similarly, consulting tables (II) and (III), we find that the large- $y$ scaling of the two-particle cut momentum parameterization (3.15) is given by,

$$
\text { y-scaling: } \quad M_{n}^{\text {tree }}(y, t) \sim\left(y^{2} / t\right)^{m} y^{2\left(h_{1}-h_{0}\right)} .
$$

From tables (II) and (III), we observe that under the loop-momentum parametrization for the triple cut (3.10),

$$
t \text {-scaling: } \quad M_{n}^{\text {tree }}(t) \sim t^{m} t^{-2 h_{0}} \text {. }
$$


This indicates that all the scalings are related up to universal helicity dependent factors. Were these observed relations to hold on very general grounds, the non-trivial cancellations in the Feynman diagrams in one of the scalings would necessarily imply non-trivial cancellations in the other scalings. In fact, this turns out to be the case, as we now argue.

To establish the above scaling relations, we write the scattering amplitude (5.1) in an abstract form making the helicity weights manifest. The tree-level scattering amplitudes are rational functions in all spinor variables. Using momentum conservation, $l_{1}=l+k_{i_{1}}+$ $\ldots+k_{i_{n-2}}$, we trade momentum $l_{1}$ for $l_{0}$, absorbing the spinor weight of the leg carrying momentum $l_{1}$ into factors of $\left\langle l_{0} l_{1}\right\rangle$. This allows us to write an amplitude with the legs carrying the momentum $l_{0}$ and $l_{1}$ with the corresponding helicities $h_{0}$ and $h_{1}$ as

$$
M_{n}^{\text {tree }}\left(l_{0}^{h_{0}},-l_{1}^{h_{1}}, i_{1}, \ldots, i_{n-2}\right)=\left\langle l_{0} l_{1}\right\rangle^{-2 h_{1}} \frac{N_{s^{\prime}}(\lambda, \tilde{\lambda})}{D_{s^{\prime \prime}}(\lambda, \tilde{\lambda})},
$$

where $N_{s^{\prime}}(\lambda, \tilde{\lambda})$ and $D_{s^{\prime \prime}}(\lambda, \tilde{\lambda})$ are polynomials in spinors $\lambda$ and $\tilde{\lambda}$, satisfying $\left(l_{0}\right)_{a \dot{a}}=\lambda_{a} \tilde{\lambda}_{\dot{a}}$ and we have suppressed the dependence on external momenta. The polynomials $N_{s^{\prime}}(\lambda, \tilde{\lambda})$ and $D_{s^{\prime \prime}}(\lambda, \tilde{\lambda})$ have definite helicity weight — the number of $\lambda \mathrm{s}$ minus the number of $\tilde{\lambda}_{\mathrm{s}}$ denoted by subscripts $s^{\prime}$ and $s^{\prime \prime}$. For the total amplitude to have proper helicity weight with respect to the $\lambda$ and $\tilde{\lambda}$, the weights $s^{\prime}$ and $s^{\prime \prime}$ must be related to the helicities by,

$$
s^{\prime}-s^{\prime \prime}=2\left(h_{1}-h_{0}\right) \text {. }
$$

In the scaling limits considered above, the polynomials are dominated by the highest degree monomials in the $\lambda$ and $\tilde{\lambda}$ variables,

$$
\begin{aligned}
N_{s^{\prime}}(\lambda, \tilde{\lambda}) & \sim \tilde{\lambda}_{\dot{a}_{1}} \cdots \tilde{\lambda}_{\dot{a}_{r^{\prime}}} \lambda_{b_{1}} \cdots \lambda_{b_{r^{\prime}+s^{\prime}}} N^{\dot{a}_{1} \ldots \dot{a}_{r^{\prime}} b_{1} \ldots b_{r^{\prime}+s^{\prime}}} \\
D_{s^{\prime \prime}}(\lambda, \tilde{\lambda}) & \sim \tilde{\lambda}_{\dot{a}_{1}} \cdots \tilde{\lambda}_{\dot{a}_{r^{\prime \prime}}} \lambda_{b_{1}} \cdots \lambda_{b_{r^{\prime \prime}+s^{\prime \prime}}} D^{\dot{a}_{1} \ldots \dot{a}_{r^{\prime \prime}} b_{1} \ldots b_{r^{\prime \prime}+s^{\prime \prime}}}
\end{aligned}
$$

A priori, the values of $r^{\prime}$ and $r^{\prime \prime}$ can be functions of the number of external legs and their helicities.

This general form of the amplitude may now be probed in the various scaling limits. For the shift $\left[l_{0},-l_{1}\right\rangle$ of the amplitude eq. (5.1) we have the scaling,

$$
\left[l_{0},-l_{1}\right\rangle: \quad M_{n}^{\text {tree }}(z) \sim z^{r^{\prime}-r^{\prime \prime}} \equiv z^{m}
$$

where we introduced the variable $m=r^{\prime}-r^{\prime \prime}$ to match eq. (15.2). Applying the $t$ and $y$ scalings to eqs. (5.6) and (5.8) immediately gives us eqs. (5.5) and (5.4). Similarly we obtain 
the flipped $z$-shift result (5.3) . Notice that the value of $m$ does not follow from this analysis and has to be derived by other means.

The relations between the various scalings thus hold on very general grounds and are applicable to a wide variety of cases including amplitudes with general matter content. In our analysis we used that the monomials in eq. (5.8) do not vanish in the scaling limits. As will be discussed elsewhere, this always holds for the scaling limits discussed here.

The $z$-scaling for the shift $[-,+\rangle$ in table【has been proven rigorously for all $n$ in ref. [32]. Direct comparison with this then allows for an easy proof of the corresponding $(-,+)$ case of the $y$ and $t$ scaling. Because the shift $[+,-\rangle$ is the flipped version of the shift $[-,+\rangle$, the two are related by the constraints of spinor weight. This then gives a proof of the large- $z$ scaling of the shift $[+,-\rangle$ in table $\square$ for all $n$. In turn this can be used to prove the $(+,-)$ cases in table II and table 【I, as well. The $[ \pm, \pm\rangle$ and $( \pm, \pm)$ cases, however, remain unproven beyond ten points for non-MHV amplitudes. For MHV amplitudes, the supersymmetry identity (2.11) allows us to apply the $[-,+\rangle$ proof to these cases as well [32].

These results demonstrate that the bubble-triangle cancellations occur whenever all tree amplitudes composing a cut have loop-momentum helicity configurations $( \pm, \mp)$. It also shows that if the $[+,+\rangle$ and $[-,-\rangle$ entries in table I are valid for any number of legs, the bubble-triangle cancellations also hold for any number of legs for the other helicity configurations of the cut legs.

\section{B. Heuristic relation between cancellations and factorization properties}

In addition, the bubble-triangle cancellations may be understood heuristically as a consequence of the stringent factorization properties of one-loop amplitudes. As any intermediate momentum $K^{\mu} \equiv k_{i}^{\mu}+\ldots+k_{i+r+1}^{\mu}$ goes on-shell $\left(K^{2} \rightarrow 0\right)$, a one-loop amplitude factorizes into lower-point amplitudes (along with a universal "factorization function" for infrared singular amplitudes [48]). As we demonstrated through ten points, for all helicities, no graviton amplitudes can have tensor triangle or bubble integrals with more than six or four powers of loop momentum in the numerators, respectively. Therefore, in all factorization limits of higher-point amplitudes we cannot encounter these integral functions or the results of reducing them to scalar integrals. Moreover, the same type of argument holds for factorizations 
where two of the external momenta become collinear ${ }^{5}$ or where one of the external momenta becomes soft. Although this does not constitute a proof ${ }^{6}$ that the unwanted tensor triangle and bubble integrals cannot appear, we know of no counterexample, in either gravity or non-abelian gauge theory where this type of factorization bootstrap argument has failed to produce the correct result at one loop.

Indeed, this argument is similar to the one used in ref. [7] to propose the no-triangle hypothesis for the $\mathcal{N}=8$ theory. In that case, there were no bubble or triangle integrals at all at lower points, predicting that bubble or triangle integrals should not appear at higher points as well.

\section{CONCLUSIONS}

In this paper we studied cancellations in pure gravity one-loop amplitudes, pointing to the existence of novel ultraviolet cancellations in generic gravity theories at higher loops. At one loop, without the novel cancellations, the two-derivative coupling of gravity would imply that under integral reductions of $n$-point amplitudes we naively would obtain bubble integrals with up to $n+2$ powers of loop momentum in their numerators. Similarly, we would obtain triangle integrals with up to $n+3$ powers of loop momentum in their numerators. Instead, under more careful scrutiny, we found that the triangle and bubble integrals resulting from integral reductions have no more than six or four powers of loop momentum, respectively.

By comparing the pure gravity case to $\mathcal{N}$-extended supergravity in various examples, we disentangled the supersymmetric cancellations in these theories from the ones which are generic to gravity. Assuming the universality of the gravity cancellations, one-loop $\mathcal{N} \geq 5$-extended supergravity amplitudes should be cut-constructible [14] using only fourdimensional momenta in the cuts. That is, they are completely determined by their absorptive parts. Similarly, the "no-triangle hypothesis" of the $\mathcal{N}=8$ theory [7, 9] may be thought of as a consequence of the combination of the pure gravity cancellations and supersymmetric cancellations. It is interesting to note that conventional superspace power counting is

\footnotetext{
5 Although there is no kinematic pole for collinear limits with real momenta, there is a universal phase singularity [6] or equivalently universal behavior under factorization with complex momenta.

${ }^{6}$ In principle, functions can be present which have no poles in any channel. An example of such a function which may occur in abelian gauge theories, may be found in eq. (14) of ref. [49].
} 
sensitive to only the latter types of cancellations.

The unitarity method, together with a new spinor-based integration method [24], allows us to link directly cancellations occurring in one-loop amplitudes to cancellations in tree-level amplitudes. This follows an early version of scaling arguments, used to imply that bubble integrals should not appear in $\mathcal{N}=8$ supergravity amplitudes [9]. Our approach allows us to determine straightforwardly the maximum powers of loop momentum that appear in the triangle and bubble integrals, including all feed downs from integral reductions. Although we have not constructed a complete proof of one-loop cancellations for all possible gravity amplitudes, we numerically demonstrated their existence up to ten points for all pure gravity helicity amplitudes, and analytically for special helicity configurations for all $n$. We have also outlined a proof for all contributions to triangle and bubble integrals whose cuts are composed of tree amplitudes with opposite graviton helicities on the legs carrying loop momentum.

The cancellations discussed here are connected to recently identified properties of gravity tree amplitudes. In the usual Lagrangian formulation an infinite set of vertices are needed to construct the scattering amplitudes. This may be contrasted with on-shell recursion relations [31] which remarkably construct all gravity tree amplitudes in $D=4$, starting only from three vertices. This property has been recently proposed as a means of classifying theories [34]. For the recursion relations to hold cancellations under certain large complex deformations of the amplitudes are necessary. The existence of these cancellations are rather obscure in Feynman diagrams. A proof of the cancellations required to have valid onshell recursion relations has recently been given in ref. [32]. Here we showed that related cancellations exist at one loop.

Unitarity implies lower-loop cancellations necessarily induce higher-loop cancellations. In particular, as shown in fig. 6(a), the cancellations in the $(L+2)$-point one-loop amplitude appearing in the $L$-particle cut of the $L$-loop amplitude, necessarily imply the existence of cancellations in the $L$-loop amplitude. Moreover, as indicated in fig. 6(b) any one-loop sub-amplitude isolated by cuts necessarily must have the cancellations found in our oneloop investigations. In ref. [2], these cuts, together with the one-loop no-triangle hypothesis, were used to suggest that $\mathcal{N}=8$ supergravity may be ultraviolet finite. These cancellations, as well as others not implied by the no-triangle hypothesis, were then confirmed by explicit calculation at three loops [3]. Similarly, the results of this paper suggest that novel 


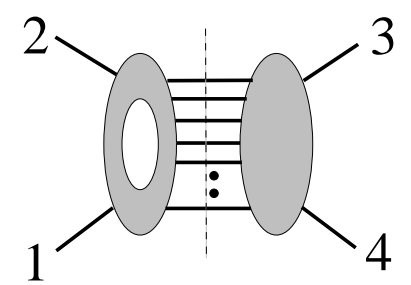

(a)

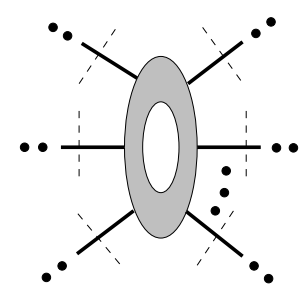

(b)

FIG. 6: From the unitarity cuts, cancellations in one-loop subamplitudes imply higher-loop cancellations. The cut (a) is an $L$-particle cut of an $L$-loop amplitude, isolating a one-loop amplitude on the left side of the cut. Diagram (b) denotes a generalized cut that isolates a particular one-loop subamplitude. If a leg is external to the entire amplitude, it should not be cut. In ref. [2], these cuts were used to argue that in $\mathcal{N}=8$ supergravity the one-loop no-triangle hypothesis implies the existence of non-trivial cancellation at higher-loop orders.

cancellations at higher loops should exist even in non-supersymmetric theories, though the cancellations will not be as strong as for $\mathcal{N}=8$ supergravity.

In order to check this, it would be rather useful to carry out explicit higher-loop studies of gravity theories. Of course, in pure gravity a two-loop divergence does exist [16], but an open question is to determine the critical dimension in which the divergences first appear, with and without matter, as the loop order increases. It would also be helpful to translate any higher-loop cancellations into the language of effective actions. For example, for the case of $\mathcal{N}=8$ supergravity, at three loops, 14 powers of external momenta can be extracted from the numerators of all loop momentum integrals, giving a contribution to the effective action of the generic form $\mathcal{D}^{6} R^{4}$ multiplied by integrals, instead of the generic form $\mathcal{D}^{4} R^{4}$, which would have been obtained if additional cancellations had not been present [3]. Here $R^{4}$ denotes an $\mathcal{N}=8$ supersymmetric contraction of Riemann tensors [13], and $\mathcal{D}$ denotes a generic space-time covariant derivative, with Lorentz indices contracted appropriately.

An important calculation that will help to answer the question of whether $\mathcal{N}=8$ supergravity is finite, and to further constrain possible superspace explanations, is to evaluate the four-point four-loop $\mathcal{N}=8$ amplitude. This would tell us whether it has the same power counting as $\mathcal{N}=4$ super-Yang-Mills theory, as already established at one [6, 7, 9, 50, 51], two [19] and three loops [3]. The four-loop calculation should be feasible, though non-trivial, following the same methods as used at three loops in ref. [3]. If $\mathcal{N}=8$ supergravity can 
be shown to be finite, an obvious question is whether there are other such theories. The absence of bubble integrals at one loop hints that theories with $\mathcal{N} \geq 5$ supersymmetries are candidate ultraviolet finite theories. One may wonder whether finiteness in gravity theories is connected to the possible existence of topological string theory descriptions [26]. Recent developments in constructing such a string for gravity may be found in ref. [52].

There are a number of other issues that would be interesting to explore. It would be important to complete a proof that any one-loop amplitude in any theory based on the Einstein-Hilbert action coupled to matter has no more than four powers of loop momentum in the numerators of bubble integrals, and no more than six powers in triangle integrals, including those generated via integral reductions. To simplify the analysis, we evaluated the cuts in $D=4$ where we have powerful spinor methods for generating amplitudes and for evaluating the resulting integrals. It would be useful to instead carry out a power counting analysis in $D=4-2 \epsilon$ dimensions to properly account for dimensional regularization. It would also be helpful to have a general one-loop analysis of the relative power counting behavior between supersymmetric and non-supersymmetric amplitudes. Finally, it would be important to re-express the cancellations in terms of effective actions.

In summary, the results of this paper point to the existence of novel loop-level cancellations in generic point-like theories of quantum gravity based on the Einstein-Hilbert action. As we showed, these cancellation are related to previously identified tree-level cancellations in gravity amplitudes under large complex deformations [31, 32, 34]. The cancellations suggest that, in general, the perturbative ultraviolet properties of quantum gravity theories may be tamer than anticipated, pointing to an improved ultraviolet behavior in the Wilsonian effective action as the cutoff is varied, independent of supersymmetry. As already suggested in refs. [2, 3] for $\mathcal{N}=8$ supergravity, the combination of these cancellations with supersymmetric ones may be sufficient to render the theory ultraviolet finite to all loop orders. Further work will be required to confirm these proposals.

\section{Acknowledgements}

We especially thank David Kosower for helpful comments and suggestions. We also thank Nathan Berkovits, Gordon Chalmers, Lance Dixon, Dave Dunbar, Renata Kallosh, Prem Kumar, Asad Naqvi, Carlos Núñez, Radu Roiban and Edward Witten for helpful comments 
and discussions. We thank Academic Technology Services at UCLA for computer support. This research was supported by the US Department of Energy under contracts DE-FG0391ER40662 and DE-AC02-76SF00515.

[1] E. Cremmer, B. Julia and J. Scherk, Phys. Lett. B 76, 409 (1978);

E. Cremmer and B. Julia, Phys. Lett. B 80, 48 (1978); Nucl. Phys. B 159, 141 (1979).

[2] Z. Bern, L. J. Dixon and R. Roiban, Phys. Lett. B 644, 265 (2007) hep-th/0611086].

[3] Z. Bern, J. J. Carrasco, L. J. Dixon, H. Johansson, D. A. Kosower and R. Roiban, Phys. Rev. Lett. 98, 161303 (2007) hep-th/0702112.

[4] G. Chalmers, hep-th/0008162;

M. B. Green, J. G. Russo and P. Vanhove, JHEP 0702, 099 (2007) hep-th/0610299、4].

[5] M. B. Green, H. Ooguri and J. H. Schwarz, arXiv:0704.0777 [hep-th].

[6] Z. Bern, L. J. Dixon, M. Perelstein and J. S. Rozowsky, Nucl. Phys. B 546, 423 (1999) hep-th/9811140.

[7] Z. Bern, N. E. J. Bjerrum-Bohr and D. C. Dunbar, JHEP 0505, 056 (2005) hep-th/0501137.

[8] N. E. J. Bjerrum-Bohr, D. C. Dunbar and H. Ita, Phys. Lett. B 621, 183 (2005) hep-th/0503102.

[9] N. E. J. Bjerrum-Bohr, D. C. Dunbar, H. Ita, W. B. Perkins and K. Risager, JHEP 0612, 072 (2006) hep-th/0610043.

[10] L. M. Brown and R. P. Feynman, Phys. Rev. 85, 231 (1952);

V. E. Asribekov, Sov. Phys. JETP 15:394 (1962);

N. Byers and C. N. Yang, Rev. Mod. Phys. 36:595 (1964);

D. B. Melrose, Nuovo Cim. 40, 181 (1965);

G. Passarino and M. J. G. Veltman, Nucl. Phys. B 160, 151 (1979);

W. L. van Neerven and J. A. M. Vermaseren, Phys. Lett. B 137, 241 (1984);

G. J. van Oldenborgh and J. A. M. Vermaseren, Z. Phys. C 46, 425 (1990).

[11] Z. Bern, L. J. Dixon and D. A. Kosower, Phys. Lett. B 302, 299 (1993) [Erratum-ibid. B 318, 649 (1993)] hep-ph/9212308;

Z. Bern, L. J. Dixon and D. A. Kosower, Nucl. Phys. B 412, 751 (1994) hep-ph/9306240;

J. Fleischer, F. Jegerlehner and O. V. Tarasov, Nucl. Phys. B 566, 423 (2000) 
hep-ph/9907327;

T. Binoth, J. P. Guillet and G. Heinrich, Nucl. Phys. B 572, 361 (2000) hep-ph/9911342;

G. Duplancic and B. Nizic, Eur. Phys. J. C 35, 105 (2004) hep-ph/0303184.

[12] H. J. Schnitzer, hep-th/0701217; arXiv:0706.0917 [hep-th].

[13] M. T. Grisaru, Phys. Lett. B 66, 75 (1977);

E. Tomboulis, Phys. Lett. B 67, 417 (1977);

S. Deser, J. H. Kay and K. S. Stelle, Phys. Rev. Lett. 38, 527 (1977);

P. S. Howe and U. Lindstrom, Nucl. Phys. B 181, 487 (1981);

R. E. Kallosh, Phys. Lett. B 99, 122 (1981);

P. S. Howe and K. S. Stelle, Int. J. Mod. Phys. A 4, 1871 (1989);

P. S. Howe and K. S. Stelle, Phys. Lett. B 554, 190 (2003) hep-th/0211279;

N. Berkovits, hep-th/0609006;

M. B. Green, J. G. Russo and P. Vanhove, Phys. Rev. Lett. 98, 131602 (2007) hep-th/0611273.

[14] Z. Bern, L. J. Dixon, D. C. Dunbar and D. A. Kosower, Nucl. Phys. B 435, 59 (1995) hep-ph/9409265.

[15] G. 't Hooft and M. J. G. Veltman, Annales Poincare Phys. Theor. A 20, 69 (1974);

G. 't Hooft, Nucl. Phys. B 62, 444 (1973).

[16] M. H. Goroff and A. Sagnotti, Phys. Lett. B 160, 81 (1985); Nucl. Phys. B 266, 709 (1986).

[17] A. E. M. van de Ven, Nucl. Phys. B 378, 309 (1992).

[18] Z. Bern, L. J. Dixon, D. C. Dunbar and D. A. Kosower, Nucl. Phys. B 425, 217 (1994) hep-ph/9403226.

[19] Z. Bern, L. J. Dixon, D. C. Dunbar, M. Perelstein and J. S. Rozowsky, Nucl. Phys. B 530, 401 (1998) hep-th/9802162.

[20] R. Britto, F. Cachazo and B. Feng, Nucl. Phys. B 725, 275 (2005) hep-th/0412103.

[21] R. Britto, E. Buchbinder, F. Cachazo and B. Feng, Phys. Rev. D 72, 065012 (2005) hep-ph/0503132;

P. Mastrolia, Phys. Lett. B 644, 272 (2007) hep-th/0611091.

[22] G. Ossola, C. G. Papadopoulos and R. Pittau, Nucl. Phys. B 763, 147 (2007) hep-ph/0609007.

[23] C. Anastasiou, R. Britto, B. Feng, Z. Kunszt and P. Mastrolia, Phys. Lett. B 645, 213 (2007) 
hep-ph/0609191;

R. Britto and B. Feng, Phys. Rev. D 75, 105006 (2007) hep-ph/0612089;

C. Anastasiou, R. Britto, B. Feng, Z. Kunszt and P. Mastrolia, JHEP 0703, 111 (2007) hep-ph/0612277.

[24] D. Forde, Phys. Rev. D 75, 125019(2007) arXiv:0704.1835 [hep-ph].

[25] Z. Bern, L. J. Dixon and D. A. Kosower, Nucl. Phys. B 513, 3 (1998) hep-ph/9708239;

Z. Bern, L. J. Dixon and D. A. Kosower, JHEP 0408, 012 (2004) hep-ph/0404293].

[26] E. Witten, Commun. Math. Phys. 252, 189 (2004) hep-th/0312171.

[27] H. Kawai, D. C. Lewellen and S. H. H. Tye, Nucl. Phys. B 269, 1 (1986).

[28] Z. Bern and A. K. Grant, Phys. Lett. B 457, 23 (1999) hep-th/9904026];

Z. Bern, A. De Freitas and H. L. Wong, Phys. Rev. Lett. 84, 3531 (2000) hep-th/9912033;

Z. Bern, Living Rev. Rel. 5, 5 (2002) [gr-qc/0206071];

N. E. J. Bjerrum-Bohr, Phys. Lett. B 560, 98 (2003) hep-th/0302131; Nucl. Phys. B 673, 41 (2003) hep-th/0305062;

N. E. J. Bjerrum-Bohr and K. Risager, Phys. Rev. D 70, 086011 (2004) hep-th/0407085;

S. Ananth and S. Theisen, arXiv:0706.1778

[29] R. Britto, F. Cachazo and B. Feng, Nucl. Phys. B 715, 499 (2005) hep-th/0412308.

[30] R. Britto, F. Cachazo, B. Feng and E. Witten, Phys. Rev. Lett. 94, 181602 (2005) hep-th/0501052].

[31] J. Bedford, A. Brandhuber, B. J. Spence and G. Travaglini, Nucl. Phys. B 721, 98 (2005) [hep-th/0502146];

F. Cachazo and P. Svrcek, hep-th/0502160;

N. E. J. Bjerrum-Bohr, D. C. Dunbar, H. Ita, W. B. Perkins and K. Risager, JHEP 0601, 009 (2006) hep-th/0509016;

A. Brandhuber, S. McNamara, B. Spence and G. Travaglini, JHEP 0703, 029 (2007) [hep-th/0701187.

[32] P. Benincasa, C. Boucher-Veronneau and F. Cachazo, hep-th/0702032.

[33] F. A. Berends, W. T. Giele and H. Kuijf, Phys. Lett. B 211, 91 (1988).

[34] P. Benincasa and F. Cachazo, arXiv:0705.4305 [hep-th].

[35] F. A. Berends, R. Kleiss, P. De Causmaecker, R. Gastmans and T. T. Wu, Phys. Lett. B 103, $124(1981)$ 
P. De Causmaecker, R. Gastmans, W. Troost and T. T. Wu, Nucl. Phys. B 206, 53 (1982);

Z. Xu, D. H. Zhang and L. Chang, TUTP-84/3-TSINGHUA;

R. Kleiss and W. J. Stirling, Nucl. Phys. B 262, 235 (1985);

J. F. Gunion and Z. Kunszt, Phys. Lett. B 161, 333 (1985);

Z. Xu, D. H. Zhang and L. Chang, Nucl. Phys. B 291, 392 (1987).

[36] M. L. Mangano and S. J. Parke, Phys. Rept. 200, 301 (1991);

L. J. Dixon, in QCD \& Beyond: Proceedings of TASI '95, ed. D. E. Soper (World Scientific, 1996) hep-ph/9601359.

[37] Z. Bern, L. J. Dixon and D. A. Kosower, arXiv:0704.2798 [hep-ph].

[38] M. T. Grisaru, H. N. Pendleton and P. van Nieuwenhuizen, Phys. Rev. D 15, 996 (1977);

M. T. Grisaru and H. N. Pendleton, Nucl. Phys. B 124, 81 (1977);

S. J. Parke and T. R. Taylor, Phys. Lett. B 157, 81 (1985) [Erratum-ibid. 174B, 465 (1986)].

[39] R. P. Feynman, F. B. Morinigo, W. G. Wagner and B. Hatfield, Reading, USA: AddisonWesley (1995) 232 p. (The advanced book program);

S. Weinberg, Phys. Rev. 138, B988 (1965);

S. Deser, Gen. Rel. Grav. 1, 9 (1970) gr-qc/0411023;

D. G. Boulware and S. Deser, Annals Phys. 89, 193 (1975).

[40] G. 't Hooft and M. J. G. Veltman, Nucl. Phys. B 44, 189 (1972).

[41] Z. Bern and D. A. Kosower, Nucl. Phys. B 379, 451 (1992);

Z. Bern, A. De Freitas, L. J. Dixon and H. L. Wong, Phys. Rev. D 66, 085002 (2002) hep-ph/0202271.

[42] W. Siegel, Phys. Lett. B 84, 193 (1979).

[43] B. S. DeWitt, Phys. Rev. 162, 1195 (1967).

[44] D. C. Dunbar and P. S. Norridge, Class. Quant. Grav. 14, 351 (1997) hep-th/9512084;

D. C. Dunbar and P. S. Norridge, Nucl. Phys. B 433, 181 (1995) hep-th/9408014.

[45] S. Deser and P. van Nieuwenhuizen, Phys. Rev. D 10, 401 (1974);

S. Deser, H. S. Tsao and P. van Nieuwenhuizen, Phys. Rev. D 10, 3337 (1974).

[46] S. J. Parke and T. R. Taylor, Phys. Rev. Lett. 56, 2459 (1986).

[47] D. C. Dunbar, J. H. Ettle and W. B. Perkins, arXiv:1011.5378 [hep-th].

[48] Z. Bern and G. Chalmers, Nucl. Phys. B 447, 465 (1995) hep-ph/9503236.

[49] Z. Bern, L. J. Dixon, D. C. Dunbar and D. A. Kosower, hep-ph/9405248. 
[50] M. B. Green, J. H. Schwarz and L. Brink, Nucl. Phys. B 198, 474 (1982).

[51] A. Nasti and G. Travaglini, arXiv:0706.0976 [hep-th].

[52] M. Abou-Zeid, C. M. Hull and L. J. Mason, hep-th/0606272;

L. J. Mason and M. Wolf, arXiv:0706.1941 [hep-th]. 